\title{
Certificateless Hybrid Signcryption ${ }^{\star}$
}

\author{
Fagen $\mathrm{Li}^{1,2,3}$, Masaaki Shirase ${ }^{3}$, and Tsuyoshi Takagi ${ }^{3}$ \\ 1 School of Computer Science and Engineering, \\ University of Electronic Science and Technology of China, Chengdu 610054, China \\ 2 Key Laboratory of Computer Networks and Information Security, \\ Xidian University, Xi'an 710071, China \\ 3 School of Systems Information Science, \\ Future University-Hakodate, Hakodate 041-8655, Japan \\ \{fagenli, shirase, takagi\}@fun.ac.jp
}

\begin{abstract}
Signcryption is a cryptographic primitive that fulfills both the functions of digital signature and public key encryption simultaneously, at a cost significantly lower than that required by the traditional signature-then-encryption approach. In this paper, we address a question whether it is possible to construct a hybrid signcryption scheme in the certificateless setting. This question seems to have never been addressed in the literature. We answer the question positively in this paper. In particular, we extend the concept of signcryption tag-KEM to the certificateless setting. We show how to construct a certificateless signcryption scheme using certificateless signcryption tag-KEM. We also give an example of certificateless signcryption tag-KEM.
\end{abstract}

Keywords: Certificateless signcryption, hybrid signcryption, signcryption tag-KEM, DEM.

\section{Introduction}

Confidentiality, integrity, non-repudiation and authentication are the important requirements for many cryptographic applications. A traditional approach to achieve these requirements is to signthen-encrypt the message. Signcryption, first proposed by Zheng [38, is a cryptographic primitive that fulfills both the functions of digital signature and public key encryption simultaneously, at a cost significantly lower than that required by the traditional signature-then-encryption approach. Several efficient signcryption schemes have been proposed since 1997 [51922 29 30 32 36 39]. The original scheme in [38] is based on the discrete logarithm problem but no security proof is given. Zheng's original scheme was only proven secure by Baek, Steinfeld, and Zheng [4] who described a formal security model in a multi-user setting. In above traditional signcryption schemes, the public key of a user is essentially a random bit string picked from a given set. So, the signcryption does not provide the authorization of the user by itself. This problem can be solved via a certificate which provides an unforgeable and trusted link between the public key and the identity of the user by the signature of a certificate authority (CA), and there is a hierarchical framework that is called public key infrastructure (PKI) to issue and manage certificates. However, the certificates management including revocation, storage, distribution and the computational cost of certificates verification is the main difficulty against traditional PKI.

To simplify key management procedures of traditional PKI, Shamir [33] proposed the concept of identity-based cryptography (IBC) in 1984. The idea of IBC is to get rid of certificates by allowing

\footnotetext{
* Full version of a paper published in The 5th Information Security Practice and Experience Conference (ISPEC 2009), LNCS 5451, pp. 112-123, Springer-Verlag, 2009.
} 
the user's public key to be any binary string that uniquely identifies the user. Examples of such strings include email addresses and IP addresses. Several practical identity-based signature (IBS) schemes have been devised since 1984 [1820], but a satisfying identity-based encryption (IBE) scheme only appeared in 2001 [10]. It was devised by Boneh and Franklin and cleverly uses bilinear maps (the Weil or Tate pairing) over supersingular elliptic curves. Subsequently, several identitybased signcryption (IBSC) schemes are also proposed [711/2 14/26/2728]. The main practical benefit of IBC is in greatly reducing the need for the public key certificates. But IBC uses a trusted third party called private key generator (PKG). The PKG generates the secret keys of all of its users, so a user can decrypt only if the PKG has given a secret key to it (so, certification is implicit), hence reduces the amount of storage and computation. On the other hand, the dependence on the PKG who can generate all users' private keys inevitably causes the key escrow problem to the IBC. For example, the PKG can decrypt any ciphertext in an IBE scheme. Equally problematical, the PKG could forge any user's signature in an IBS scheme.

To solve the key escrow problem in the IBC, Al-Riyami and Paterson [2] introduced a new paradigm called certificateless cryptography. The certificateless cryptography does not require the use of certificates and yet does not have the built-in key escrow feature of IBC. It is a model for the use of public key cryptography that is intermediate between traditional PKI and IBC. A certificateless system still makes use of a trusted third party which is called the key generating center (KGC). By way of contrast to the PKG in the IBC, the KGC does not have access to the user's private key. Instead, the KGC supplies a user with a partial private key that the KGC computes from the user's identity and a master key. The user then combines the partial private key with some secret information to generate the actual private key. The system is not identitybased, because the public key is no longer computable from a user's identity. When Alice wants to send a message to Bob in a certificateless system, she must obtain Bob's public key. However, no authentication of Bob's public key is necessary and no certificate is required. In 2008, Barbosa and Farshim [6] introduced the notion of certificateless signcryption (CLSC) and proposed an efficient scheme.

The practical way to perform secrecy communication for large messages is to use hybrid encryption that separates the encryption into two parts: one part uses public key techniques to encrypt a one-time symmetric key; the other part uses the symmetric key to encrypt the actual message. In such a construction, the public key part of the algorithm is known as the key encapsulation mechanism (KEM) while the symmetric key part is known as the data encapsulation mechanism (DEM). A formal treatment of this paradigm originates in the work of Cramer and Shoup [15]. The resulting KEM-DEM hybrid encryption paradigm has received much attention in recent years [12425. It is very attractive as it gives a clear separation between the various parts of the cipher allowing for modular design. In [1, Abe, Gennaro, and Kurosawa introduced tag-KEM which takes as input a tag in KEM. Bentahar et al. 8] extended KEM into identity-based and certificateless settings and gave generic constructions of identity-based KEM (IB-KEM) and certificateless KEM (CL-KEM). Chen et al. [13] proposed an efficient IB-KEM based on the Sakai-Kasahara key construction [31. Kiltz and Galindo [23] proposed a direct construction of IB-KEM in the standard model, based on Waters's IBE scheme [35]. Huang and Wong 21] proposed a generic construction of CL-KEM in the standard model. 
The use of hybrid techniques to build signcryption schemes has been studied by Dent [16 17]. He generalized KEM to signcryption KEM which includes an authentication in KEM. However, he only consider the insider security for authenticity. That is, if the sender's private key is exposed, an attacker is able to recover the key generated by signcryption KEM. The full insider security [3] means that (a) if the sender's private key is exposed, an attacker is still not able to recover the message from the ciphertext and (b) if the receiver's private key is exposed, an attacker is still not able to forge a ciphertext. In 2006, Bjørstad and Dent [9] showed how to built signcryption schemes using tag-KEM. However, they also only consider the insider security for authenticity and not for confidentiality. In 2008, Tan 34 proposed full insider secure signcryption KEM and tag-KEM in the standard model. Tan's schemes are insider secure for both authenticity and confidentiality. Note that the using of tag-KEM yields simpler scheme descriptions and better generic security reductions.

All the above hybrid signcryption schemes [9]16,1734] are not in the certificateless setting. In this paper, we address a question whether it is possible to construct a hybrid signcryption scheme in the certificateless setting. This question seems to have never been addressed in the literature. We answer the question positively in this paper. In particular, we extend the concept of signcryption tag-KEM to the certificateless setting. We show that a CLSC scheme can be constructed by using a certificateless signcryption tag-KEM (CLSC-TKEM) and a DEM. We also give an example of CLSC-TKEM. Our scheme is insider secure for both authenticity and confidentiality.

The rest of this paper is organized as follows. We introduce the preliminary work in Section 2 . We give the formal model of CLSC-TKEM in Section 3. We show how to construct a CLSC scheme using a CLSC-TKEM and a DEM in Section 4. An example of CLSC-TKEM is described in Section 5. Finally, the conclusions are given in Section 6 .

\section{Preliminaries}

\subsection{Certificateless Signcryption (CLSC)}

A generic CLSC scheme consists of the following six algorithms.

- Setup: This algorithm takes as input the security parameter $1^{k}$ and returns the KGC's master secret key $m s k$ and system parameters params including a master public key $m p k$ and descriptions of message space $\mathcal{M}$, ciphertext space $\mathcal{C}$ and randomness space $\mathcal{R}$. This algorithm is executed by the KGC, which publishes params.

- Extract-Partial-Private-Key: This algorithm takes as input params, msk and a user's identity $I D \in\{0,1\}^{*}$, and returns a partial private key $D_{I D}$. This algorithm is run by the $\mathrm{KGC}$, after verifying the user's identity.

- Generate-User-Keys: This algorithm takes as input params and an identity ID, and outputs a secret value $x_{I D}$ and a public key $P K_{I D}$. This algorithm is run by a user to obtain a public key and a secret value which can be used to construct a full private key. The public key is published without certification.

- Set-Private-Key: This algorithm takes as input a partial private key $D_{I D}$ and a secret value $x_{I D}$, and returns the full private key $S_{I D}$. Again, this algorithm is run by a user to construct the full private key. 
- Signcrypt: This algorithm takes as input params, a plaintext message $m \in \mathcal{M}$, the sender's full private key $S_{I D_{s}}$, identity $I D_{s}$ and public key $P K_{I D_{s}}$, and the receiver's identity $I D_{r}$ and public key $P K_{I D_{r}}$, and outputs a ciphertext $\sigma \in \mathcal{C}$.

- Unsigncrypt: This algorithm takes as input params, a ciphertext $\sigma$, the sender's identity $I D_{s}$ and public key $P K_{I D_{s}}$, and the receiver's full private key $S_{I D_{r}}$, identity $I D_{r}$ and public key $P K_{I D_{r}}$, and outputs a plaintext $m$ or a failure symbol $\perp$ if $\sigma$ is an invalid ciphertext.

We make the consistency constraint that if

$$
\sigma \leftarrow \operatorname{Signcrypt}\left(\text { params, } m, S_{I D_{s}}, I D_{s}, P K_{I D_{s}}, I D_{r}, P K_{I D_{r}}\right),
$$

then

$$
\left.m \leftarrow \text { Unsigncrypt (params, } \sigma, I D_{s}, P K_{I D_{s}}, S_{I D_{r}}, I D_{r}, P K_{I D_{r}}\right) .
$$

Barbosa and Farshim [6] defines the security notions for CLSC schemes. A CLSC scheme should satisfy confidentiality (indistinguishability against adaptive chosen ciphertext attacks (IND-CCA2)) and unforgeability (existential unforgeability against adaptive chosen messages attacks (UF-CMA)). For the stronger notion of insider security, we use the notion of strong existential unforgeability (sUF-CMA). The strong existential unforgeability means that an adversary wins if it outputs a valid message/signcryption pair $(m, \sigma)$ for identities $I D_{s}$ and $I D_{r}$ and the signcryption $\sigma$ was not returned by the signcryption oracle when queried on the message $m$. As in [11]2, we do not consider attacks targeting signcryptions where the identities of the sender and receiver are the same. That is, we disallow such queries to relevant oracles and do not accept this type of signcryption as a valid forgery.

There are two types of adversaries, Type I and Type II. A Type I adversary models an attacker which is a common user of the system and is not in possession of the KGC's master secret key. But it is able to adaptively replace users'public keys with (valid) public keys of its choice. A Type II adversary models an honest-but-curious KGC who knows the KGC's master secret key. But it cannot replace users' public keys.

For the confidentiality, we consider two games "IND-CCA2-I" and "IND-CCA2-II" where a Type I adversary $\mathcal{A}_{I}$ and a Type II adversary $\mathcal{A}_{I I}$ interact with their "challenger" in these two games, respectively. Note that the challenger keeps a history of "query-answer" while interacting with the attackers. Now we describe the two games.

IND-CCA2-I: This is the game in which $\mathcal{A}_{I}$ interacts with the "challenger":

Initial: The challenger runs $($ params, msk $) \leftarrow \operatorname{Setup}\left(1^{k}\right)$ and gives params to $\mathcal{A}_{I}$. The challenger keeps master secret key $m s k$ to itself.

Phase 1: The adversary $\mathcal{A}_{I}$ can perform a polynomially bounded number of queries in an adaptive manner.

- Extract partial private key: The adversary $\mathcal{A}_{I}$ chooses an identity $I D$. The challenger computes $D_{I D} \leftarrow$ Extract-Partial-Private-Key(params, msk, ID) and sends $D_{I D}$ to $\mathcal{A}_{I}$.

- Extract private key: The adversary $\mathcal{A}_{I}$ chooses an identity $I D$. The challenger first computes $D_{I D} \leftarrow$ Extract-Partial-Private-Key (params, msk, ID) and then computes $\left(x_{I D}, P K_{I D}\right) \leftarrow$ Generate-User-Keys (params, $I D)$. Finally, it sends the result of $S_{I D} \leftarrow \operatorname{Set}-\operatorname{Private-Key}\left(x_{I D}, D_{I D}\right)$ to $\mathcal{A}_{I}$. The adversary is not allowed to query any identity for which the corresponding public 
key has been replaced. This restriction is imposed due to the fact that it is unreasonable to expect that the challenger is able to provide a full private key for a user for which it does not know the secret value.

- Request public key: The adversary $\mathcal{A}_{I}$ chooses an identity $I D$. The challenger computes $\left(x_{I D}, P K_{I D}\right) \leftarrow$ Generate-User-Keys (params, ID) and sends $P K_{I D}$ to $\mathcal{A}_{I}$.

- Replace public key: $\mathcal{A}_{I}$ may replace a public key $P K_{I D}$ with a value chosen by it.

- Signcryption queries: The adversary $\mathcal{A}_{I}$ chooses a $m$, a sender's identity $I D_{s}$ and a receiver's identity $I D_{r}$, the challenger finds $S_{I D_{s}}$ from its "query-answer" list, computes $\sigma \leftarrow$ Signcrypt (params, $m, S_{I D_{s}}, I D_{s}, P K_{I D_{s}}, I D_{r}, P K_{I D_{r}}$ ), and returns $\sigma$ to $\mathcal{A}_{I}$. Note that, it is possible that the challenger is not aware of the sender's secret value, if the associated public key has been replaced. In this case, we require the adversary to provide it. We disallow queries where $I D_{s}=I D_{r}$.

- Unsigncryption queries: $\mathcal{A}_{I}$ chooses a $\sigma$, a sender's identity $I D_{s}$ and a receiver's identity $I D_{r}$, the challenger finds $S_{I D_{r}}$ from its "query-answer" list, computes Unsigncrypt (params, $\sigma$, $\left.I D_{s}, P K_{I D_{s}}, S_{I D_{r}}, I D_{r}, P K_{I D_{r}}\right)$, and returns the result to $\mathcal{A}_{I}$. The result is either a plaintext message $m$ or $\perp$. Note that, it is possible that the challenger is not aware of the receiver's secret value, if the associated public key has been replaced. In this case, we require the adversary to provide it. We also disallow queries where $I D_{s}=I D_{r}$.

Challenge: The adversary $\mathcal{A}_{I}$ decides when Phase 1 ends. $\mathcal{A}_{I}$ generates two equal length plaintexts $\left(m_{0}, m_{1}\right)$, a sender's identity $I D_{s}^{*}$, and a receiver's identity $I D_{r}^{*}$ on which it wishes to be challenged. Note that $I D_{r}^{*}$ should not be queried to extract a private key in Phase 1 . Note also that $I D_{r}^{*}$ cannot be equal to an identity for which both the public key has been replaced and the partial private key has been extracted. The challenger picks a random bit $\delta$ from $\{0,1\}$, computes $\sigma^{*} \leftarrow \operatorname{Signcrypt}\left(\right.$ params, $m_{\delta}, S_{I D_{s}^{*}}, I D_{s}^{*}, P K_{I D_{s}^{*}}, I D_{r}^{*}, P K_{I D_{r}^{*}}$ ), and returns $\sigma^{*}$ to $\mathcal{A}_{I}$.

Phase 2: The adversary $\mathcal{A}_{I}$ can ask a polynomially bounded number of queries adaptively again as in Phase 1 . The same rule is applied here: $\mathcal{A}_{I}$ cannot extract the private key for $I D_{r}^{*}$. $\mathcal{A}_{I}$ cannot extract the partial private key for $I D_{r}^{*}$ if the public key of this identity has been replaced before the challenge phase. In addition, $\mathcal{A}_{I}$ cannot make a unsigncryption query on $\sigma^{*}$ under $I D_{s}^{*}$ and $I D_{r}^{*}$, unless the public key $P K_{I D_{s}^{*}}$ or $P K_{I D_{r}^{*}}$ has been replaced after the challenge phase.

Guess: $\mathcal{A}_{I}$ produces a bit $\delta^{\prime}$ and wins the game if $\delta^{\prime}=\delta$.

The advantage of $\mathcal{A}_{I}$ is defined to be

$$
\operatorname{Adv}_{\mathrm{CLSC}}^{\mathrm{IND}-\mathrm{CCA} 2-\mathrm{I}}\left(\mathcal{A}_{I}\right)=\left|2 \operatorname{Pr}\left[\delta^{\prime}=\delta\right]-1\right|,
$$

where $\operatorname{Pr}\left[\delta^{\prime}=\delta\right]$ denotes the probability that $\delta^{\prime}=\delta$.

IND-CCA2-II: This is the game in which $\mathcal{A}_{I I}$ interacts with the "challenger":

Initial: The challenger runs (params, $m s k) \leftarrow \operatorname{Setup}\left(1^{k}\right)$ and gives both params and $m s k$ to $\mathcal{A}_{I I}$.

Phase 1: The adversary $\mathcal{A}_{I I}$ can perform a polynomially bounded number of queries in an adaptive manner. Note that we do not need Extract partial private key since $\mathcal{A}_{I I}$ can computes partial private keys by itself.

- Extract private key: Same to the IND-CCA2-I game.

- Request public key: Same to the IND-CCA2-I game. 
- Signcryption queries: Same to the IND-CCA2-I game.

- Unsigncryption queries: Same to the IND-CCA2-I game.

Challenge: The adversary $\mathcal{A}_{I I}$ decides when Phase 1 ends. $\mathcal{A}_{I I}$ generates two equal length plaintexts $\left(m_{0}, m_{1}\right)$, a sender's identity $I D_{s}^{*}$, and a receiver's identity $I D_{r}^{*}$ on which it wishes to be challenged. $I D_{r}^{*}$ should not be queried to extract a private key in Phase 1 . The challenger picks a random bit $\delta$ from $\{0,1\}$, computes $\sigma^{*} \leftarrow \operatorname{Signcrypt}\left(\right.$ params, $m_{\delta}, S_{I D_{s}^{*}}, I D_{s}^{*}, P K_{I D_{s}^{*}}, I D_{r}^{*}, P K_{I D_{r}^{*}}$, and returns $\sigma^{*}$ to $\mathcal{A}_{I I}$.

Phase 2: The adversary $\mathcal{A}_{I I}$ can ask a polynomially bounded number of queries adaptively again as in Phase 1. $\mathcal{A}_{I I}$ cannot extract the private key for $I D_{r}^{*}$. In addition, $\mathcal{A}_{I I}$ cannot make a unsigncryption query on $\sigma^{*}$ under $I D_{s}^{*}$ and $I D_{r}^{*}$, unless the public key $P K_{I D_{s}^{*}}$ or $P K_{I D_{r}^{*}}$ has been replaced after the challenge phase.

Guess: $\mathcal{A}_{I I}$ produces a bit $\delta^{\prime}$ and wins the game if $\delta^{\prime}=\delta$.

The advantage of $\mathcal{A}_{I I}$ is defined to be

$$
\operatorname{Adv}_{\mathrm{CLSC}}^{\mathrm{IND}-\mathrm{CCA} 2-\mathrm{II}}\left(\mathcal{A}_{I I}\right)=\left|2 \operatorname{Pr}\left[\delta^{\prime}=\delta\right]-1\right|,
$$

where $\operatorname{Pr}\left[\delta^{\prime}=\delta\right]$ denotes the probability that $\delta^{\prime}=\delta$.

Definition 1. A CLSC scheme is said to be IND-CCA2-I secure (resp. IND-CCA2-II secure) if there is no probabilistic polynomial time $(P P T)$ adversary $\mathcal{A}_{I}$ (resp. $\mathcal{A}_{I I}$ ) which wins IND-CCA2-I (resp. IND-CCA2-II) with non-negligible advantage. A CLSC scheme is said to be IND-CCA2 secure if it is both IND-CCA2-I secure and IND-CCA2-II secure.

Notice that the adversary is allowed to extract the private key of $I D_{s}^{*}$ in the IND-CCA2-I and IND-CCA2-II games. This condition corresponds to the stringent requirement of insider security for confidentiality of signcryption [3. On the other hand, it ensures the forward security of the scheme, i.e. confidentiality is preserved in case the sender's private key becomes compromised.

For the strong existential unforgeability, we consider two games "sUF-CMA-I" and "sUF-CMA-II" where a Type I adversary $\mathcal{F}_{I}$ and a Type II adversary $\mathcal{F}_{I I}$ interact with their "challenger" in these two games, respectively. Note that the challenger keeps a history of "query-answer" while interacting with the attackers. These two games are described as follows.

sUF-CMA-I: This is the game in which $\mathcal{F}_{I}$ interacts with the "challenger":

Initial: The challenger runs $($ params, $m s k) \leftarrow \operatorname{Setup}\left(1^{k}\right)$ and gives params to $\mathcal{F}_{I}$. The challenger keeps master secret key msk to itself.

Attack: The adversary $\mathcal{F}_{I}$ performs a polynomially bounded number of queries just like in the IND-CCA2-I game.

Forgery: $\mathcal{F}_{I}$ produces a quaternion $\left(m^{*}, \sigma^{*}, I D_{s}^{*}, I D_{r}^{*}\right)$. Note that $I D_{s}^{*}$ should not be queried to extract a private key. Note also that $I D_{s}^{*}$ cannot be equal to an identity for which both the public key has been replaced and the partial private key has been extracted. In addition, $\sigma^{*}$ was not returned by the signcryption oracle on the input $\left(m^{*}, I D_{s}^{*}, I D_{r}^{*}\right)$ during Attack stage. $\mathcal{F}_{I}$ wins the game if the result of Unsigncrypt (params, $\left.\sigma^{*}, I D_{s}^{*}, P K_{I D_{s}^{*}}, S_{I D_{r}^{*}}, I D_{r}^{*}, P K_{I D_{r}^{*}}\right)$ is not the $\perp$ symbol.

The advantage of $\mathcal{F}_{I}$ is defined as the probability that it wins.

sUF-CMA-II: This is the game in which $\mathcal{F}_{I I}$ interacts with the "challenger": 
Initial: The challenger runs $($ params, $m s k) \leftarrow \operatorname{Setup}\left(1^{k}\right)$ and gives both params and $m s k$ to $\mathcal{F}_{I I}$.

Attack: The adversary $\mathcal{F}_{I I}$ performs a polynomially bounded number of queries just like in the IND-CCA2-II game.

Forgery: $\mathcal{F}_{I I}$ produces a quaternion $\left(m^{*}, \sigma^{*}, I D_{s}^{*}, I D_{r}^{*}\right) . I D_{s}^{*}$ should not be queried to extract a private key. In addition, $\sigma^{*}$ was not returned by the signcryption oracle on the input $\left(m^{*}, I D_{s}^{*}, I D_{r}^{*}\right)$ during Attack stage. $\mathcal{F}_{I I}$ wins the game if the result of Unsigncrypt (params, $\sigma^{*}, I D_{s}^{*}, P K_{I D_{s}^{*}}, S_{I D_{r}^{*}}$, $\left.I D_{r}^{*}, P K_{I D_{r}^{*}}\right)$ is not the $\perp$ symbol.

The advantage of $\mathcal{F}_{I I}$ is defined as the probability that it wins.

Definition 2. A CLSC scheme is said to be sUF-CMA-I secure (resp. sUF-CMA-II secure) if there is no PPT adversary $\mathcal{F}_{I}$ (resp. $\mathcal{F}_{I I}$ ) which wins sUF-CMA-I (resp. sUF-CMA-II) with non-negligible advantage. A CLSC scheme is said to be sUF-CMA secure if it is both sUF-CMA-I secure and sUF-CMA-II secure.

Note that the adversary is allowed to extract the private key of $I D_{r}^{*}$ in the above definition. Again, this condition corresponds to the stringent requirement of insider security for signcryption [3].

\subsection{Date Encapsulation Mechanism (DEM)}

A DEM is a symmetric encryption scheme which consists of the following two algorithms.

- Enc: This algorithm takes as input $1^{k}$, a key $K$ and a message $m \in\{0,1\}^{*}$, and outputs a ciphertext $c \in\{0,1\}^{*}$, where $K \in \mathcal{K}_{\text {DEM }}$ is a key in the given key space, and $m$ is a bit string of arbitrary length. We denote this as $c \leftarrow \operatorname{Enc}(K, m)$.

- Dec: This algorithm takes as input a key $K$ and a ciphertext $c$, and outputs the message $m \in\{0,1\}^{*}$ or a symbol $\perp$ to indicate that the ciphertext is invalid.

For the purposes of this paper, it is only required that a DEM is secure with respect to indistinguishability against passive attackers (IND-PA). Formally, this security notion is captured by the following game played between a PPT adversary $\mathcal{A}$ and a challenger.

Initial: $\mathcal{A}$ runs on input $1^{k}$ and submits two equal length messages, $m_{0}$ and $m_{1}$.

Challenge: The challenger chooses a random key $K \in \mathcal{K}_{\text {DEM }}$ as well as a random bit $\lambda \in\{0,1\}$, and sends $c^{*} \leftarrow \operatorname{Enc}\left(K, m_{\lambda}\right)$ to $\mathcal{A}$ as a challenge ciphertext.

Guess: The adversary $\mathcal{A}$ produces a bit $\lambda^{\prime}$ and wins the game if $\lambda^{\prime}=\lambda$.

The advantage of $\mathcal{A}$ is defined to be

$$
A d v_{\mathrm{DEM}}^{\mathrm{IND}-\mathrm{PA}}(\mathcal{A})=\left|2 \operatorname{Pr}\left[\lambda^{\prime}=\lambda\right]-1\right|
$$

where $\operatorname{Pr}\left[\lambda^{\prime}=\lambda\right]$ denotes the probability that $\lambda^{\prime}=\lambda$.

Definition 3. A DEM is said to be IND-PA secure if there is no PPT adversary $\mathcal{A}$ which wins the above game with non-negligible advantage. 


\section{Certificateless Signcryption Tag-KEM (CLSC-TKEM)}

In this section, we extend the concept of signcryption tag-KEM to the certificateless setting. We give the formal definition for certificateless signcryption tag-KEM (CLSC-TKEM).

\subsection{Generic Scheme}

A generic CLSC-TKEM consists of the following seven algorithms.

- Setup: Same to CLSC described in Section 2.

- Partial-Private-Key-Extract: Same to CLSC described in Section 2.

- Generate-User-Keys: Same to CLSC described in Section 2.

- Set-Private-Key: Same to CLSC described in Section 2.

- Sym: This is symmetric key generation algorithm which takes as input the params, the sender's full private key $S_{I D_{s}}$, identity $I D_{s}$ and public key $P K_{I D_{s}}$, the receiver's identity $I D_{r}$ and public key $P K_{I D_{r}}$, and outputs a symmetric key $K$ together with internal state information $\omega$. Here $K \in \mathcal{K}_{\text {CLSC-TKEM }}$ is a key in the space of possible session keys at a given security level. We denote this as $(K, \omega) \leftarrow \operatorname{Sym}\left(\right.$ params, $\left.S_{I D_{s}}, I D_{s}, P K_{I D_{s}}, I D_{r}, P K_{I D_{r}}\right)$.

- Encap: This is key encapsulation algorithm which takes as input the state information $\omega$ and

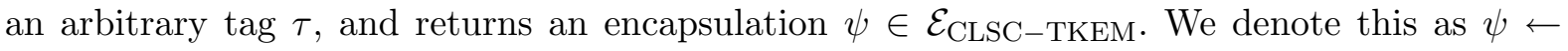
$\operatorname{Encap}(\omega, \tau)$.

- Decap: This is decapsulation algorithm which takes as input the params, an encapsulation $\psi$, a tag $\tau$, the sender's identity $I D_{s}$ and public key $P K_{I D_{s}}$, the receiver's full private key $S_{I D_{r}}$, identity $I D_{r}$ and public key $P K_{I D_{r}}$, and outputs a key $K$ or a special symbol $\perp$ indicating invalid encapsulation. We denote this as $K \leftarrow \operatorname{Decap}\left(\right.$ params, $\left.\psi, \tau, I D_{s}, P K_{I D_{s}}, S_{I D_{r}}, I D_{r}, P K_{I D_{r}}\right)$.

We make the consistency constraint that if

$$
(K, \omega) \leftarrow \operatorname{Sym}\left(\text { params, } S_{I D_{s}}, I D_{s}, P K_{I D_{s}}, I D_{r}, P K_{I D_{r}}\right) \text { and } \psi \leftarrow \operatorname{Encap}(\omega, \tau),
$$

then

$$
K \leftarrow \operatorname{Decap}\left(\text { params, } \psi, \tau, I D_{s}, P K_{I D_{s}}, S_{I D_{r}}, I D_{r}, P K_{I D_{r}}\right)
$$

\subsection{Security Notions}

A CLSC-TKEM should satisfy confidentiality and unforgeability. To define the security notions for CLSC-TKEM, we simply adapt the security notions of CLSC into the TKEM framework.

Again there are two types of adversary against a CLSC-TKEM: Type I and Type II. A Type I adversary models an attacker which is a common user of the system and is not in possession of the KGC's master secret key. But it is able to adaptively replace users'public keys with (valid) public keys of its choice. A Type II adversary models an honest-but-curious KGC who knows the KGC's master secret key. But it cannot replace users' public keys.

For the confidentiality, we consider two games "IND-CCA2-I" and "IND-CCA2-II" where a Type I adversary $\mathcal{A}_{I}$ and a Type II adversary $\mathcal{A}_{I I}$ interact with their "challenger" in these two games, respectively. Note that the challenger keeps a history of "query-answer" while interacting with the attackers. Now we describe the two games. 
IND-CCA2-I: This is the game in which $\mathcal{A}_{I}$ interacts with the "challenger":

Initial: The challenger runs (params,msk) $\leftarrow \operatorname{Setup}\left(1^{k}\right)$ and gives params to $\mathcal{A}_{I}$. The challenger keeps master secret key $m s k$ to itself.

Phase 1: The adversary $\mathcal{A}_{I}$ can perform a polynomially bounded number of queries in an adaptive manner.

- Extract partial private key: Same to CLSC's IND-CCA2-I game described in Section 2 .

- Extract private key: Same to CLSC's IND-CCA2-I game described in Section 2 ,

- Request public key: Same to CLSC's IND-CCA2-I game described in Section 2.

- Replace public key: Same to CLSC's IND-CCA2-I game described in Section 2 .

- Symmetric key generation queries: $\mathcal{A}_{I}$ chooses a sender's identity $I D_{s}$ and a receiver's identity $I D_{r}$. The challenger finds $S_{I D_{s}}$ from its "query-answer" list and runs $(K, \omega) \leftarrow \operatorname{Sym}($ params, $S_{I D_{s}}, I D_{s}, P K_{I D_{s}}, I D_{r}, P K_{I D_{r}}$ ). The challenger then stores the value $\omega$ (hidden from the view of the adversary, and overwriting any previously stored values), and sends the symmetric key $K$ to $\mathcal{A}_{I}$. Note that, it is possible that the challenger is not aware of the sender's secret value, if the associated public key has been replaced. In this case, we require the adversary to provide it. We disallow queries where $I D_{s}=I D_{r}$.

- Key encapsulation queries: $\mathcal{A}_{I}$ produces an arbitrary tag $\tau$. The challenger checks whether there exists a stored value $\omega$. If not, it returns $\perp$ and terminates. Otherwise it erases the value from storage and returns $\psi \leftarrow \operatorname{Encap}(\omega, \tau)$ to $\mathcal{A}_{I}$.

- Key decapsulation queries: The adversary $\mathcal{A}_{I}$ chooses a sender's identity $I D_{s}$, a receiver's identity $I D_{r}$, an encapsulation $\psi$, and a tag $\tau$. The challenger finds $S_{I D_{r}}$ from its "queryanswer" list and sends the result of Decap (params, $\left.\psi, \tau, I D_{s}, P K_{I D_{s}}, S_{I D_{r}}, I D_{r}, P K_{I D_{r}}\right)$ to $\mathcal{A}_{I}$. Note that, it is possible that the challenger is not aware of the receiver's secret value, if the associated public key has been replaced. In this case, we require the adversary to provide it. We also disallow queries where $I D_{s}=I D_{r}$.

Challenge: The adversary $\mathcal{A}_{I}$ decides when Phase 1 ends. $\mathcal{A}_{I}$ generates a sender's identity $I D_{s}^{*}$ and a receiver's identity $I D_{r}^{*}$ on which it wishes to be challenged. Note that $I D_{r}^{*}$ should not be queried to extract a private key in Phase 1 . Note also that $I D_{r}^{*}$ cannot be equal to an identity for which both the public key has been replaced and the partial private key has been extracted. The challenger computes $\left(K_{1}, \omega^{*}\right) \leftarrow \operatorname{Sym}\left(\right.$ params, $\left.S_{I D_{s}^{*}}, I D_{s}^{*}, P K_{I D_{s}^{*}}, I D_{r}^{*}, P K_{I D_{r}^{*}}\right)$. Then the challenger chooses $K_{0} \leftarrow \mathcal{K}_{\text {CLSC-TKEM }}$ and a bit $b \in\{0,1\}$ randomly, and sends $K_{b}$ to $\mathcal{A}_{I}$. When $\mathcal{A}_{I}$ receives $K_{b}$, it may ask the same queries as previously. Then $\mathcal{A}_{I}$ generates a tag $\tau^{*}$. The challenger computes $\psi^{*} \leftarrow \operatorname{Encap}\left(\omega^{*}, \tau^{*}\right)$ and sends it to $\mathcal{A}_{I}$ as a challenge encapsulation.

Phase 2: The adversary $\mathcal{A}_{I}$ can ask a polynomially bounded number of queries adaptively again as in Phase 1 . The same rule is applied here: $\mathcal{A}_{I}$ cannot extract the private key for $I D_{r}^{*} . \mathcal{A}_{I}$ cannot extract the partial private key for $I D_{r}^{*}$ if the public key of this identity has been replaced before the challenge phase. In addition, $\mathcal{A}_{I}$ cannot make a decapsulation query on $\left(K_{b}, \psi^{*}\right)$ under $I D_{s}^{*}$ and $I D_{r}^{*}$, unless the public key $P K_{I D_{s}^{*}}$ or $P K_{I D_{r}^{*}}$ has been replaced after the challenge phase.

Guess: The adversary $\mathcal{A}_{I}$ produces a bit $b^{\prime}$ and wins the game if $b^{\prime}=b$.

The advantage of $\mathcal{A}_{I}$ is defined to be

$$
\operatorname{Adv}_{\mathrm{CLSC}-\mathrm{TKEM}}^{\mathrm{IND}-\mathrm{CCA} 2-\mathrm{I}}\left(\mathcal{A}_{I}\right)=\left|2 \operatorname{Pr}\left[b^{\prime}=b\right]-1\right|,
$$


where $\operatorname{Pr}\left[b^{\prime}=b\right]$ denotes the probability that $b^{\prime}=b$.

IND-CCA2-II: This is the game in which $\mathcal{A}_{I I}$ interacts with the "challenger":

Initial: The challenger runs (params, msk) $\leftarrow \operatorname{Setup}\left(1^{k}\right)$ and gives both params and $m s k$ to $\mathcal{A}_{I I}$.

Phase 1: The adversary $\mathcal{A}_{I I}$ can perform a polynomially bounded number of queries in an adaptive manner. Note that we do not need Extract partial private key since $\mathcal{A}_{I I}$ can computes partial private keys by itself.

- Extract private key: Same to CLSC's IND-CCA2-I game described in Section 2

- Request public key: Same to CLSC's IND-CCA2-I game described in Section 2.

- Symmetric key generation queries: Same to CLSC-TKEM's IND-CCA2-I game described in Section 3 ,

- Key encapsulation queries: Same to CLSC-TKEM's IND-CCA2-I game described in Section 3 .

- Key decapsulation queries: Same to CLSC-TKEM's IND-CCA2-I game described in Section 3.

Challenge: The adversary $\mathcal{A}_{I I}$ decides when Phase 1 ends. $\mathcal{A}_{I I}$ generates a sender's identity $I D_{s}^{*}$ and a receiver's identity $I D_{r}^{*}$ on which it wishes to be challenged. Note that $I D_{r}^{*}$ should not be queried to extract a private key in Phase 1 . The challenger runs $\left(K_{1}, \omega^{*}\right) \leftarrow \operatorname{Sym}\left(\right.$ params, $S_{I D_{s}^{*}}, I D_{s}^{*}$, $\left.P K_{I D_{s}^{*}}, I D_{r}^{*}, P K_{I D_{r}^{*}}\right)$. Then the challenger chooses $K_{0} \leftarrow \mathcal{K}_{\mathrm{CLSC}-\mathrm{TKEM}}$ and a bit $b \in\{0,1\}$ randomly, and sends $K_{b}$ to $\mathcal{A}_{I}$. When $\mathcal{A}_{I I}$ receives $K_{b}$, it may ask the same queries as previously. Then $\mathcal{A}_{I I}$ generates a tag $\tau^{*}$. The challenger computes $\psi^{*} \leftarrow \operatorname{Encap}\left(\omega^{*}, \tau^{*}\right)$ and sends it to $\mathcal{A}_{I I}$ as a challenge encapsulation.

Phase 2: The adversary $\mathcal{A}_{I I}$ can ask a polynomially bounded number of queries adaptively again as in Phase 1. $\mathcal{A}_{I I}$ cannot extract the private key for $I D_{r}^{*}$. In addition, $\mathcal{A}_{I I}$ cannot make a decapsulation query on $\left(K_{b}, \psi^{*}\right)$ under $I D_{s}^{*}$ and $I D_{r}^{*}$, unless the public key $P K_{I D_{s}^{*}}$ or $P K_{I D_{r}^{*}}$ has been replaced after the challenge phase.

Guess: The adversary $\mathcal{A}_{I I}$ produces a bit $b^{\prime}$ and wins the game if $b^{\prime}=b$.

The advantage of $\mathcal{A}_{I I}$ is defined to be

$$
\operatorname{Adv} \underset{\mathrm{CLSC}-\mathrm{TKEM}}{\mathrm{IND}}\left(\mathcal{A}_{I I}\right)=\left|2 \operatorname{Pr}\left[b^{\prime}=b\right]-1\right|,
$$

where $\operatorname{Pr}\left[b^{\prime}=b\right]$ denotes the probability that $b^{\prime}=b$.

Definition 4. A CLSC-TKEM scheme is said to be IND-CCA2-I secure (resp. IND-CCA2-II secure) if there is no PPT adversary $\mathcal{A}_{I}$ (resp. $\mathcal{A}_{I I}$ ) which wins IND-CCA2-I (resp. IND-CCA2-II) with non-negligible advantage. A CLSC-TKEM scheme is said to be IND-CCA2 secure if it is both IND-CCA2-I secure and IND-CCA2-II secure.

Notice that the adversary is allowed to extract the private key of $I D_{s}^{*}$ in the IND-CCA2-I and IND-CCA2-II games. This condition corresponds to the stringent requirement of insider security for confidentiality of signcryption [3. On the other hand, it ensures the forward security of the scheme, i.e. confidentiality is preserved in case the sender's private key becomes compromised.

For the strong existential unforgeability, we consider two games "SUF-CMA-I" and "sUF-CMA-II" where a Type I adversary $\mathcal{F}_{I}$ and a Type II adversary $\mathcal{F}_{I I}$ interact with their "challenger" in these 
two games, respectively. Note that the challenger keeps a history of "query-answer" while interacting with the attackers. Now we describe the two games.

sUF-CMA-I: This is the game in which $\mathcal{F}_{I}$ interacts with the "challenger":

Initial: The challenger runs $($ params, msk $) \leftarrow \operatorname{Setup}\left(1^{k}\right)$ and gives params to $\mathcal{F}_{I}$. The challenger keeps master secret key msk to itself.

Attack: The adversary $\mathcal{F}_{I}$ performs a polynomially bounded number of queries just like in the CLSC-TKEM's IND-CCA2-I game.

Forgery: $\mathcal{F}_{I}$ produces a quaternion $\left(\tau^{*}, \psi^{*}, I D_{s}^{*}, I D_{r}^{*}\right)$. Note that $I D_{s}^{*}$ should not be queried to extract a private key. Note also that $I D_{s}^{*}$ cannot be equal to an identity for which both the public key has been replaced and the partial private key has been extracted. In addition, $\psi^{*}$ was not returned by the key encapsulation oracle on the input $\left(\tau^{*}, I D_{s}^{*}, I D_{r}^{*}\right)$ during Attack stage. $\mathcal{F}_{I}$ wins the game if the result of Decap (params, $\left.\psi^{*}, \tau^{*}, I D_{s}^{*}, P K_{I D_{s}^{*}}, S_{I D_{r}^{*}}, I D_{r}^{*}, P K_{I D_{r}^{*}}\right)$ is not the $\perp$ symbol.

The advantage of $\mathcal{F}_{I}$ is defined as the probability that it wins.

sUF-CMA-II: This is the game in which $\mathcal{F}_{I I}$ interacts with the "challenger":

Initial: The challenger runs $($ params, $m s k) \leftarrow \operatorname{Setup}\left(1^{k}\right)$ and gives both params and $m s k$ to $\mathcal{F}_{I I}$.

Attack: The adversary $\mathcal{F}_{I I}$ performs a polynomially bounded number of queries just like in the CLSC-TKEM's IND-CCA2-II game.

Forgery: $\mathcal{F}_{I I}$ produces a quaternion $\left(\tau^{*}, \psi^{*}, I D_{s}^{*}, I D_{r}^{*}\right)$. I $D_{s}^{*}$ should not be queried to extract a private key. In addition, $\psi^{*}$ was not returned by the key encapsulation oracle on the input $\left(\tau^{*}, I D_{s}^{*}, I D_{r}^{*}\right)$ during Attack stage. $\mathcal{F}_{I I}$ wins the game if the result of Decap $\left(\right.$ params $, \psi^{*}, \tau^{*}, I D_{s}^{*}$, $\left.P K_{I D_{s}^{*}}, S_{I D_{r}^{*}}, I D_{r}^{*}, P K_{I D_{r}^{*}}\right)$ is not the $\perp$ symbol.

The advantage of $\mathcal{F}_{I I}$ is defined as the probability that it wins.

Definition 5. A CLSC-TKEM scheme is said to be sUF-CMA-I secure (resp. sUF-CMA-II secure) if there is no PPT adversary $\mathcal{F}_{I}$ (resp. $\mathcal{F}_{I I}$ ) which wins sUF-CMA-I (resp. sUF-CMA-II) with nonnegligible advantage. A CLSC-TKEM scheme is said to be sUF-CMA secure if it is both sUF-CMA-I secure and sUF-CMA-II secure.

Note that the adversary is allowed to extract the private key of $I D_{r}^{*}$ in the above definition. Again, this condition corresponds to the stringent requirement of insider security for signcryption [3].

\section{Certificateless Hybrid Signcryption}

We can combine a CLSC-TKEM with a DEM to form a CLSC scheme. We describe it in Figure 1. Note that the tag is the ciphertext output by the DEM. Such construction yields simpler scheme descriptions and better generic security reductions.

We give the security results for such construction in Theorems 1 and 2 .

Theorem 1. Let CLSC be a certificateless hybrid signcryption scheme constructed from a CLSCTKEM and a DEM. If the CLSC-TKEM is IND-CCA2 secure and the DEM is IND-PA secure, 
CLSC.Setup: On input $1^{k}$ :

1. $($ params,$m s k) \leftarrow$ CLSC-TKEM. $\operatorname{Setup}\left(1^{k}\right)$

2. Output the system parameters params and the master secret key msk

CLSC.Partial-Private-Key-Extract: On input the params, $m s k$, and an identity $I D \in\{0,1\}^{*}$ :

1. $D_{I D} \leftarrow$ CLSC-TKEM.Partial-Private-Key-Extract (params, msk, ID)

2. Output the partial private key $D_{I D}$ of the identity $I D$

CLSC.Generate-User-Keys: On input the params and an identity $I D \in\{0,1\}^{*}$ :

1. $\left(x_{I D}, P K_{I D}\right) \leftarrow$ CLSC-TKEM. Generate-User-Keys $($ params,$I D)$

2. Output the secret value $x_{I D}$ and the public key $P K_{I D}$ of the identity $I D$

CLSC.Set-Private-Key: On input the partial private key $D_{I D}$ and the secret value $x_{I D}$ :

1. $S_{I D} \leftarrow$ CLSC-TKEM.Set-Private-Key $\left(D_{I D}, x_{I D}\right)$

2. Output the full private key $S_{I D}$

CLSC.Signcrypt: On input the params, a message $m \in\{0,1\}^{*}$, the sender's full private key $S_{I D_{s}}$, identity $I D_{s}$ and public key $P K_{I D_{s}}$, the receiver's identity $I D_{r}$ and public key $P K_{I D_{r}}$ :

1. $(K, \omega) \leftarrow$ CLSC-TKEM.Sym $\left(\right.$ params, $\left.S_{I D_{s}}, I D_{s}, P K_{I D_{s}}, I D_{r}, P K_{I D_{r}}\right)$

2. $c \leftarrow \operatorname{DEM} . \operatorname{Enc}(K, m)$

3. $\psi \leftarrow \operatorname{CLSC}-\mathrm{TKEM} . \operatorname{Encap}(\omega, c)$

4. Output the ciphertext $\sigma \leftarrow(\psi, c)$

CLSC.Unsigncrypt: On input the params, a ciphertext $\sigma$, the sender's identity $I D_{s}$ and public key $P K_{I D_{s}}$, the receiver's full private key $S_{I D_{r}}$, identity $I D_{r}$ and public key $P K_{I D_{r}}$ :

1. $K \leftarrow$ CLSC-TKEM. Decap $\left(\right.$ params $\left., \psi, c, I D_{s}, P K_{I D_{s}}, S_{I D_{r}}, I D_{r}, P K_{I D_{r}}\right)$

2. If $K=\perp$, then output $\perp$ and stop

3. $m \leftarrow \mathrm{DEM} \cdot \operatorname{Dec}(K, c)$

4. Output the message $m$

Fig. 1. Certificateless hybrid signcryption

then $C L S C$ is IND-CCA2 secure. In particular, we have

$$
\operatorname{Adv}_{\mathrm{CLSC}}^{\mathrm{IND}-\mathrm{CCA} 2-\mathrm{i}}(\mathcal{A}) \leq 2 \operatorname{Adv}_{\mathrm{CLSC}-\mathrm{TKEM}}^{\mathrm{IND}-\mathrm{CCA} 2-\mathrm{i}}\left(\mathcal{B}_{1}\right)+\operatorname{Adv}_{\mathrm{DEM}}^{\mathrm{IND}-\mathrm{PA}}\left(\mathcal{B}_{2}\right)
$$

where $i \in\{I, I I\}$

Proof. See the appendix A.

Theorem 2. Let CLSC be a certificateless hybrid signcryption scheme constructed from a CLSCTKEM and a DEM. If the CLSC-TKEM is sUF-CMA secure, then CLSC is sUF-CMA secure. In 
particular, we have

$$
\operatorname{Adv}_{\mathrm{CLSC}}^{\mathrm{sUF}-\mathrm{CMA}-\mathrm{i}}(\mathcal{F}) \leq \operatorname{Adv}_{\mathrm{CLSC}-\mathrm{TKEM}}^{\mathrm{sUF}-\mathrm{CMA}-\mathrm{i}}(\mathcal{B}),
$$

where $i \in\{I, I I\}, \operatorname{Adv}_{\mathrm{CLSC}}^{\mathrm{sUF}-\mathrm{CMA}-\mathrm{i}}(\mathcal{F})$ is the advantage of the sUF-CMA adversary against CLSC, and $\operatorname{Adv}_{\mathrm{CLSC}-\mathrm{TKEM}}^{\mathrm{SUF}-\mathrm{CMA}-\mathrm{B})}$ is the advantage of the resulting sUF-CMA adversary against CLSCTKEM.

Proof. See the appendix B.

\section{An Example of CLSC-TKEM}

The Barbosa-Farshim CLSC scheme [6] fits the new generic framework. Here we give an example of CLSC-TKEM based on the Barbosa-Farshim scheme. If we combine the CLSC-TKEM with a DEM as Figure 1, we can get a scheme that is very similar to the Barbosa-Farshim scheme. Since the Barbosa-Farshim scheme uses the bilinear pairings, we describe some basic knowledge about bilinear pairings in the appendix $\mathrm{C}$.

\section{$5.1 \quad$ CLSC-TKEM}

The CLSC-TKEM consists of the following seven algorithms.

- Setup: Define $G_{1}, G_{2}$ and $\hat{e}$ as in appendix C. Let $H_{1}, H_{2}, H_{3}$, and $H_{4}$ be four cryptographic hash functions where $H_{1}:\{0,1\}^{*} \rightarrow G_{1}, H_{2}:\{0,1\}^{*} \rightarrow\{0,1\}^{n}, H_{3}:\{0,1\}^{*} \rightarrow G_{1}$, and $H_{4}:\{0,1\}^{*} \rightarrow G_{1}$. Here $n$ is the key length of a DEM. Let $P$ be a generator of $G_{1}$. The PKG chooses a master secret key $s \in Z_{q}^{*}$ randomly and computes $P_{p u b} \leftarrow s P$. The PKG publishes system parameters $\left\{G_{1}, G_{2}, n, \hat{e}, P, P_{p u b}, H_{1}, H_{2}, H_{3}, H_{4}\right\}$ and keeps the master key $s$ secret.

- Partial-Private-Key-Extract: Given an identity $I D \in\{0,1\}^{*}$, the PKG computes $Q_{I D} \leftarrow$ $H_{1}(I D)$ and returns the partial private key $D_{I D} \leftarrow s Q_{I D}$.

- Generate-User-Keys: A user with identity $I D$ chooses a random element $x_{I D}$ from $Z_{q}$ as the secret value, and sets $P K_{I D} \leftarrow x_{I D} P$ as the public key.

- Set-Private-Key: Given a partial private key $D_{I D}$ and a secret value $x_{I D}$, this algorithm returns the full private key $S_{I D} \leftarrow\left(x_{I D}, D_{I D}\right)$.

- Sym: Given the sender's full private key $S_{I D_{s}}$, identity $I D_{s}$ and public key $P K_{I D_{s}}$, the receiver's identity $I D_{r}$ and public key $P K_{I D_{r}}$, this algorithm works as follows.

1. Choose $r \in Z_{q}^{*}$ randomly.

2. Compute $U=r P$ and $T \leftarrow \hat{e}\left(P_{p u b}, Q_{I D_{r}}\right)^{r}$.

3. Compute $K \leftarrow H_{2}\left(U, T, r P K_{I D_{r}}, I D_{r}, P K_{I D_{r}}\right)$.

4. Output $K$ and set $\omega \leftarrow\left(r, U, S_{I D_{s}}, I D_{s}, P K_{I D_{s}}, I D_{r}, P K_{I D_{r}}\right)$.

- Encap: Given the state information $\omega$ and an arbitrary tag $\tau$, this algorithm works as follows.

1. Compute $H \leftarrow H_{3}\left(U, \tau, I D_{s}, P K_{I D_{s}}\right)$.

2. Compute $H^{\prime} \leftarrow H_{4}\left(U, \tau, I D_{s}, P K_{I D_{s}}\right)$.

3. Compute $W \leftarrow D_{I D_{s}}+r H+x_{I D_{s}} H^{\prime}$

4. Output $\psi \leftarrow(U, W)$ 
- Decap: Given the the sender's identity $I D_{s}$ and public key $P K_{I D_{s}}$, the receiver's full private key $S_{I D_{r}}$, identity $I D_{r}$ and public key $P K_{I D_{r}}$, an encapsulation $\psi$ and a tag $\tau$, this algorithm works as follows.

1. Compute $H \leftarrow H_{3}\left(U, \tau, I D_{s}, P K_{I D_{s}}\right)$.

2. Compute $H^{\prime} \leftarrow H_{4}\left(U, \tau, I D_{s}, P K_{I D_{s}}\right)$.

3. If $\hat{e}\left(P_{p u b}, Q_{I D_{s}}\right) \hat{e}(U, H) \hat{e}\left(P K_{I D_{s}}, H^{\prime}\right)=\hat{e}(P, W)$, compute $T=\hat{e}\left(D_{I D_{r}}, U\right)$ and output the $K \leftarrow H_{2}\left(U, T, x_{I D_{r}} U, I D_{r}, P K_{I D_{r}}\right)$. Otherwise, output symbol $\perp$.

\subsection{Security}

We give the security results for the CLSC-TKEM in Theorems 3 and 4

Theorem 3. In the random oracle model, the above CLSC-TKEM is IND-CCA2 secure under the assumption that the gap bilinear Diffie-Hellman problem is intractable.

Proof. See the appendix D.

Theorem 4. In the random oracle model, the above CLSC-TKEM is sUF-CMA secure under the assumption that the GDH' problem is intractable.

Proof. See the appendix E.

\section{Conclusions}

In this paper, we extended the concept of signcryption tag-KEM to the certificateless setting. We showed that a certificateless signcryption scheme can be constructed by combining a certificateless signcryption tag-KEM with a DEM. To show that our framework is reasonable, we also gave an example of certificateless signcryption tag-KEM based on the Barbosa-Farshim certificateless signcryption scheme.

\section{Acknowledgements}

This work is supported by the National Natural Science Foundation of China (Grant Nos. 60673075, 60803133 and 60873233), the Key Laboratory of Computer Networks and Information Security of Xidian University (2008CNIS-02), and the Youth Science and Technology Foundation of UESTC. Fagen Li is supported by the JSPS postdoctoral fellowship for research in Japan.

\section{References}

1. M. Abe, R. Gennaro, and K. Kurosawa. Tag-KEM/DEM: a new framework for hybrid encryption. Journal of Cryptology, Vol. 21, No. 1, pp. 97-130, 2008.

2. S.S. Al-Riyami and K.G. Paterson. Certificateless public key cryptography. In Advances in CryptologyASIACRYPT 2003, LNCS 2894, pp. 452-474, Springer-Verlag, 2003.

3. J.H. An, Y. Dodis, T. Rabin. On the security of joint signature and encryption. In Advances in CryptologyEUROCRYPT 2002, LNCS 2332, pp. 83-107, Springer-Verlag, 2002. 
4. J. Baek, R. Steinfeld, and Y. Zheng. Formal proofs for the security of signcryption. Journal of Cryptology, Vol. 20, No 2, pp. 203-235, 2007.

5. F. Bao and R.H. Deng. A signcryption scheme with signature directly verifiable by public key. In Public Key Cryptography-PKC'98, LNCS 1431, pp. 55-59, Springer-Verlag, 1998.

6. M. Barbosa and P. Farshim. Certificateless signcryption. In ACM Symposium on Information, Computer and Communications Security-ASIACCS 2008, pp. 369-372, Tokyo, Japan, 2008.

7. P.S.L.M. Barreto, B. Libert, N. McCullagh, and J.J. Quisquater. Efficient and provably-secure identity-based signatures and signcryption from bilinear maps. In Advances in Cryptology-ASIACRYPT 2005, LNCS 3788, pp. 515-532, Springer-Verlag, 2005.

8. K. Bentahar, P. Farshim, J. Malone-Lee, and N.P. Smart. Generic constructions of identity-based and certificateless KEMs. Journal of Cryptology, Vol. 21, No 2, pp. 178-199, 2008.

9. T.E. Bjørstad and A.W. Dent. Building better signcryption schemes with tag-KEMs. In Public Key CryptographyPKC 2006, LNCS 3958, pp. 491-507, Springer-Verlag, 2006.

10. D. Boneh and M. Franklin. Identity-based encryption from the weil pairing. In Advances in Cryptology-CRYPTO 2001, LNCS 2139, pp. 213-229, Springer-Verlag, 2001.

11. X. Boyen. Multipurpose identity-based signcryption: a swiss army knife for identity-based cryptography. In $A d-$ vances in Cryptology-CRYPTO 2003, LNCS 2729, pp. 383-399, Springer-Verlag, 2003.

12. L. Chen and J. Malone-Lee. Improved identity-based signcryption. In Public Key Cryptography-PKC 2005, LNCS 3386, pp. 362-379, Springer-Verlag, 2005.

13. L. Chen, Z. Cheng, J. Malone-Lee, N.P. Smart. Efficient ID-KEM based on the Sakai-Kasahara key construction. IEE Proceedings-Information Security, Vol. 153, No 1, pp. 19-26, 2006.

14. S.S.M. Chow, S.M. Yiu, L.C.K. Hui, and K.P. Chow. Efficient forward and provably secure ID-based signcryption scheme with public verifiability and public ciphertext authenticity. In Information Security and Cryptology-ICISC 2003, LNCS 2971, pp. 352-369, Springer-Verlag, 2004.

15. R. Cramer and V. Shoup. Design and analysis of practical public-key encryption schemes secure against adaptive chosen ciphertext attack. SIAM Journal on Computing, Vol. 33, No. 1, pp. 167-226, 2003.

16. A.W. Dent. Hybrid signcryption schemes with outsider security. In Information Security-ISC 2005, LNCS 3650, pp. 203-217, Springer-Verlag, 2005.

17. A.W. Dent. Hybrid signcryption schemes with insider security. In Information Security and Privacy-ACISP 2005, LNCS 3574, pp. 253-266, Springer-Verlag, 2005.

18. A. Fiat and A. Shamir. How to prove yourself: practical solutions to identification and signature problems. In Advances in Cryptology-CRYPTO'86, LNCS 263, pp. 186-194, Springer-Verlag, 1986.

19. C. Gamage, J. Leiwo, and Y. Zheng. Encrypted message authentication by firewalls. In Public Key CryptographyPKC'99, LNCS 1560, pp. 69-81, Springer-Verlag, 1999.

20. L. Guillou and J.J. Quisquater. A "Paradoxical" Identity-based signature scheme resulting from zero-knowledge. In Advances in Cryptology-CRYPTO'88, LNCS 403, pp. 216-231, Springer-Verlag, 1988.

21. Q. Huang and D.S. Wong. Generic certificateless key encapsulation mechanism. In Information Security and Privacy-ACISP 200\%, LNCS 4586, pp. 215-229, Springer-Verlag, 2007.

22. H.Y. Jung, D.H. Lee, J.I. Lim, and K.S. Chang. Signcryption schemes with forward secrecy. In Information Security Application-WISA 2001, pp. 463-475, Seoul, Korea, 2001.

23. E. Kiltz and D. Galindo. Direct chosen-ciphertext secure identity-based key encapsulation without random oracles. In Information Security and Privacy-ACISP 2006, LNCS 4058, pp. 336-347, Springer-Verlag, 2006.

24. E. Kiltz. Chosen-ciphertext secure key-encapsulation based on gap hashed diffie-hellman. In Public Key Cryptography-PKC 2007, LNCS 4450, pp. 282-297, Springer-Verlag, 2007.

25. K. Kurosawa and Y. Desmedt. A new paradigm of hybrid encryption scheme. In Advances in Cryptology-CRYPTO 2004, LNCS 3152, pp. 426-442, Springer-Verlag, 2004.

26. B. Libert and J.J. Quisquater. A new identity based signcryption schemes from pairings. In 2003 IEEE Information Theory Workshop, pp. 155-158, Paris, France, 2003.

27. F. Li, Y. Hu, and Chuanrong Zhang. An identity-based signcryption scheme for multi-domain ad hoc networks. In Applied Cryptography and Network Security-ACNS 2007, LNCS 4521, pp. 373-384, Springer-Verlag, 2007.

28. J. Malone-Lee. Identity based signcryption. Cryptology ePrint Archive, Report 2002/098, 2002.

29. J. Malone-Lee and W. Mao. Two birds one stone: signcryption using RSA. In Topics in Cryptology-CT-RSA 2003, LNCS 2612, pp. 211-226, Springer-Verlag, 2003. 
30. Y. Mu and V. Varadharajan. Distributed signcryption. In Progress in Cryptology-INDOCRYPT 2000, LNCS 1977, pp. 155-164, Springer-Verlag, 2000.

31. R. Sakai and M. Kasahara. ID based cryptosystems with pairing on elliptic curve. Cryptology ePrint Archive, Report 2003/054, 2003.

32. M. Seo and K. Kim. Electronic funds transfer protocol using domain-verifiable signcryption scheme. In Information Security and Cryptology-ICISC'99, LNCS 1787, pp. 269-277, Springer-Verlag, 1999.

33. A. Shamir. Identity-based cryptosystems and signature schemes. In Advances in Cryptology-CRYPTO'84, LNCS 196, pp. 47-53, Springer-Verlag, 1984.

34. C.H. Tan. Insider-secure signcryption KEM/tag-KEM schemes without random oracles. In The Third International Conference on Availability, Reliability and Security-ARES 2008, pp. 1275-1281, Barcelona, Spain, 2008.

35. B. Waters. Efficient identity-based encryption without random oracles. In Advances in Cryptology-EUROCRYPT 2005, LNCS 3494, pp. 114-127, Springer-Verlag, 2005,

36. D.H. Yum and P.J. Lee. New signcryption schemes based on KCDSA. In Information Security and CryptologyICISC 2001, LNCS 2288, pp. 305-317, Springer-Verlag, 2002.

37. V. Shoup. OAEP reconsidered. In Advances in Cryptology-CRYPTO 2001, LNCS 2139, pp. 239-259, SpringerVerlag, 2001.

38. Y. Zheng. Digital signcryption or how to achieve cost (signature \& encryption) « cost (signature) + cost(encryption). In Advances in Cryptology-CRYPTO'97, LNCS 1294, pp. 165-179, Springer-Verlag, 1997.

39. Y. Zheng and H. Imai. How to construct efficient signcryption schemes on elliptic curves. Information Processing Letters, Vol. 68, No.5, pp. 227-233, 1998.

\section{Appendix}

\section{A Proof of Theorem 1}

Proof. Our proof strategy is as follows. We define a sequence Game ${ }_{0}, \mathrm{Game}_{1}, \mathrm{Game}_{2}$ of modified attack games. The only difference between games is how the environment responds to $\mathcal{A}$ 's oracle queries.

Let $\sigma^{*} \leftarrow\left(\psi^{*}, c^{*}\right)$ be the challenge ciphertext submitted to $\mathcal{A}$ by its challenge oracle that encrypts either $m_{0}$ or $m_{1}$ according to a bit $b$. Let $K^{*}$ denote the symmetric key used by the challenge oracle in the generation of the challenge ciphertext, or alternatively, the decapsulation of $\psi^{*}$ using the identities $I D_{s}^{*}$ and $I D_{r}^{*}$ that are chosen by the adversary. For any $i=0,1,2$, we let $S_{i}$ be the event that $\delta^{\prime}=\delta$ in game Game ${ }_{i}$, where $\delta$ is the bit chosen by $\mathcal{A}$ 's challenge oracle and $\delta^{\prime}$ is the bit output by $\mathcal{A}$. This probability is taken over the random choices of $\mathcal{A}$ and those of $\mathcal{A}$ 's oracles.

We will use the following useful Lemma 1 from [37].

Lemma 1. Let $E, E^{\prime}$, and $F$ be events defined on a probability space such that $\operatorname{Pr}[E \wedge \neg F]=$ $\operatorname{Pr}\left[E^{\prime} \wedge \neg F\right]$. Then we have

$$
\left|\operatorname{Pr}[E]-\operatorname{Pr}\left[E^{\prime}\right]\right| \leq \operatorname{Pr}[F]
$$

Game $_{0}$ : We simulate the view of the adversary in a real attack by running the suitable key generation algorithms and using the resulting keys to respond to $\mathcal{A}$ 's queries. So the view of $\mathcal{A}$ is the same as it would be in a real attack. Therefore, we have

$$
\left|\operatorname{Pr}\left[S_{0}\right]-\frac{1}{2}\right|=\frac{1}{2} \operatorname{Adv}_{\mathrm{CLSC}}^{\mathrm{IND}-\mathrm{CCA} 2-\mathrm{i}}(\mathcal{A}),
$$

where $i \in\{I, I I\}$.

Game $_{1}$ : In this game, we slightly modify how the unsigncryption oracle responds to queries from $\mathcal{A}$. When a sender' identity $I D_{s}$, a receiver's identity $I D_{r}$, and $(\psi, c)$ is presented to the 
unsigncryption oracle after the invocation of the challenge signcryption oracle, if $I D_{s}=I D_{s}^{*}$, $I D_{r}=I D_{r}^{*}$ and $\psi=\psi^{*}$, and in the case of a Type I adversary, the public keys of $I D_{s}^{*}$ and $I D_{r}^{*}$ have not been replaced, then the unsigncryption oracle does not use the genuine unsigncryption procedure for the hybrid scheme, instead it uses the key $K^{*}$ to decrypt $c$ and returns the result to the adversary $\mathcal{A}$.

Clearly this change has no impact on the adversary and so

$$
\operatorname{Pr}\left[S_{1}\right]=\operatorname{Pr}\left[S_{0}\right]
$$

Game $_{2}$ : In this game, we modify Game 1 by replacing $K^{*}$ with a random key $K^{\prime}$ from $\mathcal{K}_{\text {DEM }}$. The result then follows from the following Lemmas 2 and 3.

Lemma 2. There exists a PPT algorithm $\mathcal{B}_{1}$, whose running time is essentially the same as that of $\mathcal{A}$, such that

$$
\left|\operatorname{Pr}\left[S_{2}\right]-\operatorname{Pr}\left[S_{1}\right]\right|=\operatorname{Adv}_{\mathrm{CLSC}-\mathrm{TKEM}}^{\mathrm{IND}-\mathrm{CCA} 2-\mathrm{i}}\left(\mathcal{B}_{1}\right)
$$

where $i \in\{I, I I\}$.

Proof. To prove this we demonstrate how to construct an adversary $\mathcal{B}_{1}$ of the CLSC-TKEM to violate the IND-CCA2-I (resp. IND-CCA2-II) attack.

Adversary $\mathcal{B}_{1}$ is constructed by running adversary $\mathcal{A}$. We respond to $\mathcal{A}$ 's queries as follows.

- When $\mathcal{A}$ calls any oracle, bar its signcryption, unsigncryption and challenge signcryption oracles, $\mathcal{B}_{1}$ simply relays these queries to its own equivalent oracle.

- When $\mathcal{A}$ make a signcryption query with a sender's identity $I D_{s}$, a receiver's identity $I D_{r}$ and a plaintext $m, \mathcal{B}_{1}$ follows the steps below.

1. Make a symmetric key generation query on $\left(I D_{s}, I D_{r}\right)$ to its own symmetric key generation oracle to obtain $K$.

2. Compute $c \leftarrow$ DEM.Enc $(K, m)$.

3. Make a key encapsulation query on $c$ to its own key encapsulation oracle to obtain $\psi$.

4. Return the ciphertext $\sigma \leftarrow(\psi, c)$ to $\mathcal{A}$.

- When $\mathcal{A}$ make a unsigncryption query with a sender's identity $I D_{s}$, a receiver's identity $I D_{r}$ and a ciphertext $\sigma \leftarrow(\psi, c), \mathcal{B}_{1}$ follows the steps below.

1. Make a key decapsulation query on $\left(\psi, c, I D_{s}, I D_{r}\right)$ to its own key decapsulation oracle to obtain $K$.

2. If $K=\perp$, return $\perp$ and stop.

3. Compute $m \leftarrow \mathrm{DEM} . \operatorname{Dec}(K, c)$ and return $m$.

- When $\mathcal{A}$ calls its challenge signcryption oracle with two equal length plaintexts $m_{0}, m_{1}$, a sender's identity $I D_{s}^{*}$, and a receiver's identity $I D_{r}^{*}, \mathcal{B}_{1}$ follows the steps below.

1. Submit $I D_{s}^{*}$ and $I D_{r}^{*}$ to its challenger to obtain $K_{b}$, where $b \in\{0,1\}$.

2. Pick a random bit $\delta$ from $\{0,1\}$.

3. Compute $c^{*} \leftarrow$ DEM.Enc $\left(K_{b}, m_{\delta}\right)$.

4. Submit $c^{*}$ to its challenger to obtain $\psi^{*}$.

5. Return the ciphertext $\sigma^{*} \leftarrow\left(\psi^{*}, c^{*}\right)$ to $\mathcal{A}$. 
- To respond to $\mathcal{A}$ 's unsigncryption query for a sender's identity $I D_{s}$, a receiver's identity $I D_{r}$ and a ciphertext $\sigma \leftarrow(\psi, c)$ after $\mathcal{A}$ has queried its challenge signcryption oracle, $\mathcal{B}_{1}$ proceeds as follows.

- If $\left(I D_{s}, I D_{r}, \psi\right) \neq\left(I D_{s}^{*}, I D_{r}^{*}, \psi^{*}\right)$ then it uses the same procedure that it used before $\mathcal{A}$ 's call to its challenge signcryption oracle.

- In the case of a Type I adversary against a CLSC scheme, if $\left(I D_{s}, I D_{r}, \psi\right)=\left(I D_{s}^{*}, I D_{r}^{*}, \psi^{*}\right)$ and the public keys have been replaced, then $\mathcal{B}_{1}$ responds by calling the key decapsulation oracle provided to it by $\mathcal{A}$ with input $\left(I D_{s}^{*}, I D_{r}^{*}, \psi^{*}, c^{*}\right)$ to obtain $K$. It then uses $K$ to decrypt $c$ and relays the response to $\mathcal{A}$.

- Otherwise, $\mathcal{B}_{1}$ uses $K_{b}$ to decrypt $c$ and relays the result to $\mathcal{A}$.

At the end of the simulation, $\mathcal{A}$ outputs $\delta^{\prime}$. If $\delta^{\prime}=\delta, \mathcal{B}_{1}$ outputs $b^{\prime}=1$ indicating $K_{b}$ is the real key; otherwise it outputs $b^{\prime}=0$ indicating $K_{b}$ is a random key.

When $K_{b}$ is the real key, $\mathcal{A}$ is run exactly as it would be run in Game ${ }_{1}$. This means that

$$
\operatorname{Pr}\left[S_{1}\right]=\operatorname{Pr}\left[\delta^{\prime}=\delta \mid b=1\right]=\operatorname{Pr}\left[b^{\prime}=1 \mid b=1\right] .
$$

When $K_{b}$ is the random key, $\mathcal{A}$ is run exactly as it would be in Game 2 . This means that

$$
\operatorname{Pr}\left[S_{2}\right]=\operatorname{Pr}\left[\delta^{\prime}=\delta \mid b=0\right]=\operatorname{Pr}\left[b^{\prime}=1 \mid b=0\right] .
$$

From the definition of security for CLSC-TKEM, we have

$$
\operatorname{Adv}_{\mathrm{CLSC}-\mathrm{TKEM}}^{\mathrm{IND}-\mathrm{CCA} 2-\mathrm{i}}\left(\mathcal{B}_{1}\right)=\left|2 \operatorname{Pr}\left[b^{\prime}=b\right]-1\right|=\left|\operatorname{Pr}\left[b^{\prime}=1 \mid b=1\right]-\operatorname{Pr}\left[b^{\prime}=1 \mid b=0\right]\right| .
$$

So the result holds.

Lemma 3. There exists a PPT algorithm $\mathcal{B}_{2}$, whose running time is essentially the same as that of $\mathcal{A}$, such that

$$
\left|\operatorname{Pr}\left[S_{2}\right]-\frac{1}{2}\right|=\frac{1}{2} \operatorname{Adv}_{\mathrm{DEM}}^{\mathrm{IND}-\mathrm{PA}}\left(\mathcal{B}_{2}\right)
$$

Proof. To construct such a $\mathcal{B}_{2}$ we simply run $\mathcal{A}$ as it would be run in game $\mathrm{Game}_{2}$. We run the suitable CLSC-TKEM algorithms so we can respond to $\mathcal{A}$ 's queries before it calls its challenge signcryption oracle. When $\mathcal{A}$ calls its challenge signcryption oracle with a sender's identity $I D_{s}^{*}$, a receiver's identity $I D_{r}^{*}$, and messages $\left(m_{0}, m_{1}\right)$, we simply relay $\left(m_{0}, m_{1}\right)$ to the challenge encryption oracle of $\mathcal{B}_{2}$ to obtain $c^{*}$. We then make a symmetric key generation query and a key encapsulation query to obtain $K^{*}$ and $\psi^{*}$, respectively. We discard $K^{*}$ and return $\left(\psi^{*}, c^{*}\right)$ to $\mathcal{A}$. We continue to respond to $\mathcal{A}$ 's queries as before except if it a makes unsigncryption query on $\left(I D_{s}^{*}, I D_{r}^{*}, \psi^{*}, c\right)$ for some $c$. In this instance there are two cases:

- If we are dealing with a Type I adversary $\mathcal{A}$ of a CLSC scheme, and the public keys have been replaced, then $\mathcal{B}_{2}$ decapsulates $\left(I D_{s}^{*}, I D_{r}^{*}, \psi^{*}, c\right)$ using the provided secret key to obtain $K$, decrypts $c$ and relays the response to $\mathcal{A}$.

- Otherwise we query $\mathcal{B}_{2}$ 's decryption oracle with $c$ and relay the response to $\mathcal{A}$.

In this simulation $\mathcal{A}$ is run by $\mathcal{B}_{2}$ in exactly the same manner as the former would be run in game Game ${ }_{2} ;$ moreover, $\operatorname{Pr}\left[S_{2}\right]$ corresponds exactly to the probability that $\mathcal{B}_{2}$ correctly determines the hidden bit of its challenge encryption oracle since $\mathcal{B}_{2}$ outputs whatever $\mathcal{A}$ outputs. The result follows. 


\section{B Proof of Theorem 2}

Proof. Suppose that $\mathcal{F}$ is an adversary that breaks the CLSC scheme with probability $\operatorname{Adv}_{\mathrm{CLSC}}^{\mathrm{sUF}-\mathrm{CMA}-\mathrm{i}}(\mathcal{F})$, where $i \in\{I, I I\}$. We use this to construct an algorithm $\mathcal{B}$ that breaks the sUF-CMA-i for the CLSC-TKEM with probability at least $\operatorname{Adv}_{\mathrm{CLSC}}^{\mathrm{sUF}-\mathrm{CMA}-\mathrm{i}}(\mathcal{F})$ too.

Adversary $\mathcal{B}$ is constructed by running adversary $\mathcal{F}$. We respond to $\mathcal{F}$ 's queries as follows.

- When $\mathcal{F}$ calls any oracle, bar its signcryption and unsigncryption oracles, $\mathcal{B}$ simply relays these queries to its own equivalent oracle.

- When $\mathcal{F}$ make a signcryption query with a sender's identity $I D_{s}$, a receiver's identity $I D_{r}$ and a plaintext $m, \mathcal{B}$ follows the steps below.

1. Make a symmetric key generation query on $\left(I D_{s}, I D_{r}\right)$ to its own symmetric key generation oracle to obtain $K$.

2. Compute $c \leftarrow \mathrm{DEM} . \operatorname{Enc}(K, m)$.

3. Make a key encapsulation query on $c$ to its own key encapsulation oracle to obtain $\psi$.

4. Return the ciphertext $\sigma \leftarrow(\psi, c)$ to $\mathcal{F}$.

- When $\mathcal{F}$ make a unsigncryption query with a sender's identity $I D_{s}$, a receiver's identity $I D_{r}$ and a ciphertext $\sigma \leftarrow(\psi, c), \mathcal{B}$ follows the steps below.

1. Make a key decapsulation query on $\left(\psi, c, I D_{s}, I D_{r}\right)$ to its own key decapsulation oracle to obtain $K$.

2. If $K=\perp$, return $\perp$ and stop.

3. Compute $m \leftarrow \operatorname{DEM} . \operatorname{Dec}(K, c)$ and return $m$.

Finally, $\mathcal{F}$ outputs a forgery $\left(m^{*}, \sigma^{*}, I D_{s}^{*}, I D_{r}^{*}\right)$, where $\left(\psi^{*}, c^{*}\right) \leftarrow \sigma^{*} . \mathcal{B}$ outputs $\left(\tau^{*}, \psi^{*}, I D_{s}^{*}, I D_{r}^{*}\right)$, where $\tau^{*}=c^{*}$.

Clearly, this algorithm perfectly simulates the environment in which $\mathcal{F}$ should be running. If $\mathcal{F}$ wins the sUF-CMA-i for the CLSC, $\mathcal{B}$ have the same probability to win the sUF-CMA-i for CLSC-TKEM.

\section{Bilinear Pairings}

Let $G_{1}$ be a cyclic additive group generated by $P$, whose order is a prime $q$, and $G_{2}$ be a cyclic multiplicative group of the same order $q$. A bilinear pairing is a map $\hat{e}: G_{1} \times G_{1} \rightarrow G_{2}$ with the following properties:

1. Bilinearity: $\hat{e}(a P, b Q)=\hat{e}(P, Q)^{a b}$ for all $P, Q \in G_{1}, a, b \in Z_{q}$.

2. Non-degeneracy: There exists $P$ and $Q \in G_{1}$ such that $\hat{e}(P, Q) \neq 1$.

3. Computability: There is an efficient algorithm to compute $\hat{e}(P, Q)$ for all $P, Q \in G_{1}$.

The modified Weil pairing and the Tate pairing [10] are admissible maps of this kind. The security of our scheme described here relies on the hardness of the following problems.

Definition 6. We say the gap bilinear Diffie-Hellman (GBDH) assumption holds if the advantage of any PPT adversary as defined below is negligible.

$$
\operatorname{Adv}_{G B D H}\left(\mathcal{A}, q_{D B D H}\right)=\operatorname{Pr}\left[T=\hat{e}(P, P)^{a b c} \mid a, b, c \leftarrow Z_{q} ; T \leftarrow \mathcal{A}^{\mathcal{O}}(P, a P, b P, c P)\right]
$$


In the above equation, $\mathcal{O}$ denotes a decision bilinear Diffie-Hellman oracle which on input $(P, a P, b P, c P, T)$ outputs 1 if $T=\hat{e}(P, P)^{a b c}$ and 0 otherwise. By $q_{D B D H}$ we denote the maximum number of queries that $\mathcal{A}$ asks its decision oracle.

The following weaker assumption is implied by the above.

Definition 7. We say the computational Diffie-Hellman assumption in the presence of a decision bilinear Diffie-Hellman oracle $\left(G D H^{\prime}\right)$ holds in $G_{1}$ if the advantage of any PPT adversary as defined below is negligible.

$$
\operatorname{Adv}_{G D H^{\prime}}\left(\mathcal{A}, q_{D B D H}\right)=\operatorname{Pr}\left[Q=a b P \mid a, b \leftarrow Z_{q} ; Q \leftarrow \mathcal{A}^{\mathcal{O}}(P, a P, b P)\right]
$$

Here $\mathcal{O}$ and $q_{D B D H}$ are as in the above definition.

This assumption in turn implies:

Definition 8. We say the computational Diffie-Hellman (CDH) assumption holds in $G_{1}$ if the advantage of any PPT adversary as defined below is negligible.

$$
\operatorname{Adv}_{C D H}(\mathcal{A})=\operatorname{Pr}\left[Q=a b P \mid a, b \leftarrow Z_{q} ; Q \leftarrow \mathcal{A}(P, a P, b P)\right]
$$

\section{Proof of Theorem 3}

Proof. In the Barbosa-Farshim CLSC scheme [6], they use a weaker formulation of Type I adversary which they refer to as Type I'. In confidentiality games, the Type $\mathrm{I}^{\prime}$ adversary is not allowed to extract the partial private key of $I D_{r}^{*}$. They proved that If a CLSC scheme is IND-CCA2 secure against Type II and Type I' attackers, then it is also IND-CCA2 secure against Type I attackers. It is easy to extend this conclusion to CLSC-TKEM setting. That is, we have the following Lemma 4.

Lemma 4. If a CLSC-TKEM is IND-CCA2 secure against Type II and Type I' attackers then it is also IND-CCA2 secure against Type I attackers. In particular, we have

$$
\operatorname{Adv}_{\mathrm{CLSC}}^{\mathrm{IND}-\mathrm{CCA} 2-\mathrm{I}}(\mathcal{A}) \leq 2 \operatorname{Adv}_{\mathrm{CLSC}}^{\mathrm{IND}-\mathrm{CCA} 2-\mathrm{I}^{\prime}}\left(\mathcal{C}_{1}\right)+\operatorname{Adv}_{\mathrm{CLSC}}^{\mathrm{IND}-\mathrm{CCA} 2-\mathrm{II}}\left(\mathcal{C}_{2}\right)
$$

This theorem follows from Lemmas 4, 5 and 6.

Lemma 5. Under the GBDH assumption, no PPT attacker $\mathcal{A}$ has non-negligible advantage in winning the IND-CCA2-I' game against the above CLSC-TKEM, when all hash functions are modeled as random oracles. More precisely, there exists an algorithm $\mathcal{C}$ which uses $\mathcal{A}$ to solve the $G B D H$ problem such that:

$$
\operatorname{Adv}_{\mathrm{CLSC}}^{\mathrm{IND}-\mathrm{CCA} 2-\mathrm{I}^{\prime}}(\mathcal{A}) \leq q_{T} \operatorname{Adv}_{G B D H}\left(\mathcal{C}, q_{D}^{2}+2 q_{D} q_{2}+q_{2}\right)
$$

where $q_{T}=q_{1}+q_{P}+q_{K}+2 q_{D}+2$. Here $q_{1}, q_{2}, q_{P}, q_{K}$ and $q_{D}$ are the maximum number of queries that the adversary can ask $H_{1}$, ask $H_{2}$, extract partial private key, extract private key and make key decapsulation queries. 
Proof. The challenger $\mathcal{C}$ takes as input $(P, a P, b P, c P)$ and attempts to compute $\hat{e}(P, P)^{a b c} . \mathcal{C}$ will run $\mathcal{A}$ as a subroutine and act as $\mathcal{A}$ 's challenger in the IND-CCA2-I' game for CLSC-TKEM. During the game, $\mathcal{A}$ will consult $\mathcal{C}$ for answers to the random oracles $H_{1}, H_{2}, H_{3}$ and $H_{4}$. Roughly speaking, these answers are randomly generated, but to maintain the consistency and to avoid collision, $\mathcal{C}$ keeps three lists $L_{1}, L_{2}, L_{3}, L_{4}$ respectively to store the answers. The following assumptions are made.

1. $\mathcal{A}$ will ask for $H_{1}(I D)$ before $I D$ is used in any partial private key extraction, private key extraction, symmetric key generation, key encapsulation and key decapsulation queries.

2. Key encapsulation returned from a key encapsulation query will not be used by $\mathcal{A}$ in a key decapsulation query.

At the beginning of the game, $\mathcal{C}$ gives $\mathcal{A}$ the system parameters with $P_{\text {pub }} \leftarrow a P$. Note that $a$ is unknown to $\mathcal{C}$. This value simulates the master secret key for the KGC in the game. $\mathcal{C}$ chooses a random number $j \in\left\{1,2, \ldots, q_{T}\right\}$ and answers various oracle queries as follows.

$H_{1}$ queries: $\mathcal{A}$ asks a polynomially bounded number of $H_{1}$ queries on identities of his choice. At the $j$-th $H_{1}$ query, $\mathcal{C}$ answers by $H_{1}\left(I D_{j}\right) \leftarrow b P$ and puts $\left(I D_{j}, \perp\right)$ to list $L_{1}$. For queries $H_{1}\left(I D_{i}\right)$ with $i \neq j, \mathcal{C}$ chooses $e_{i} \in Z_{q}^{*}$ randomly, puts $\left(I D_{i}, e_{i}\right)$ in list $L_{1}$ and answers $H_{1}\left(I D_{i}\right) \leftarrow e_{i} P$.

Extract partial private key: When $\mathcal{A}$ asks a partial private key extraction query on identity $I D_{i}$, if $I D_{i}=I D_{j}$, then $\mathcal{C}$ fails and stops. If $I D_{i} \neq I D_{j}$, then the list $L_{1}$ must contain $\left(I D_{i}, e_{i}\right)$ for some $e_{i}$ (this indicates $\mathcal{C}$ previously answered $H_{1}\left(I D_{i}\right) \leftarrow e_{i} P$ on a $H_{1}$ query on $\left.I D_{i}\right) \cdot \mathcal{C}$ returns the partial private key $D_{I D_{i}} \leftarrow e_{i} a P$.

Request public key: When $\mathcal{A}$ asks a public key query on identity $I D_{i}, \mathcal{C}$ checks the list $L_{K}$, which is initially empty. If there is a tuple $\left(I D_{i}, P K_{I D_{i}}, x_{I D_{i}}\right)$, then $\mathcal{C}$ returns $P K_{I D_{i}}$. Otherwise, $\mathcal{C}$ generates a new key pair, updates the list $L_{K}$, and returns the public key.

Replace public key: On input $\left(I D_{i}, P K_{I D_{i}}\right), \mathcal{C}$ inserts/updates $L_{K}$ with tuple $\left(I D_{i}, P K_{I D_{i}}, \perp\right)$. Extract private key: When $\mathcal{A}$ asks a private key extraction query on identity $I D_{i}, \mathcal{C}$ calls $H_{1}$ on $I D_{i}$ and obtains $\left(I D_{i}, e_{i}\right)$. If $I D_{i}=I D_{j}$, then $\mathcal{C}$ fails and stops. Otherwise, $\mathcal{C}$ searches $L_{K}$ for the entry $\left(I D_{i}, P K_{I D_{i}}, x_{I D_{i}}\right)$, generating a new key pair if this does not exist, and returns $S_{I D_{i}} \leftarrow\left(x_{I D_{i}}, e_{i} a P\right)$.

$H_{3}$ Queries: When $\mathcal{A}$ asks a $H_{3}$ query on $\left(U_{i}, \tau_{i}, I D_{i}, P K_{I D_{i}}\right), \mathcal{C}$ checks if the list $L_{3}$ contains a tuple $\left(U_{i}, \tau_{i}, I D_{i}, P K_{I D_{i}}, t_{i}, t_{i} P\right)$. If such a tuple is found, $\mathcal{C}$ answers $t_{i} P$. Otherwise, $\mathcal{C}$ chooses a random value $t \in Z_{q}$, puts the $\left(U_{i}, \tau_{i}, I D_{i}, P K_{I D_{i}}, t, t P\right)$ into $L_{3}$, and returns $t P$.

$H_{4}$ Queries: When $\mathcal{A}$ asks a $H_{4}$ query on $\left(U_{i}, \tau_{i}, I D_{i}, P K_{I D_{i}}\right), \mathcal{C}$ checks if the list $L_{4}$ contains a tuple $\left(U_{i}, \tau_{i}, I D_{i}, P K_{I D_{i}}, l_{i}, l_{i} P\right)$. If such a tuple is found, $\mathcal{C}$ answers $l_{i} P$. Otherwise, $\mathcal{C}$ chooses a random value $l \in Z_{q}$, puts the $\left(U_{i}, \tau_{i}, I D_{i}, P K_{I D_{i}}, l, l P\right)$ into $L_{4}$, and returns $l P$.

$H_{2}$ queries: For each new query $\left(U_{i}, T_{i}, R_{i}, I D_{i}, P K_{I D_{i}}\right), \mathcal{C}$ proceeds as follows:

1. It checks if the decision bilinear Diffie-Hellman oracle returns 1 when queried with the tuple $\left(a P, b P, c P, T_{i}\right)$. If this is the case, $\mathcal{C}$ returns $T_{i}$ and stop.

2. $\mathcal{C}$ goes through the list $L_{2}$ with entries $\left(U_{i}, \star, R_{i}, I D_{i}, P K_{I D_{i}}, h_{i}\right)$, for different values of $h_{i}$, such that the decision bilinear Diffie-Hellman oracle returns 1 when queried on the tuple $\left(a P, b P, U_{i}, T_{i}\right)$. Note that in this case $I D_{i}=I D_{j}$. If such a tuple exists, it returns $h_{i}$ (and replaces the symbol $\star$ with $T_{i}$ ) 
3. If $\mathcal{C}$ reaches this point of execution, it returns a random $h$ and updates the list $L_{2}$, which is initially empty, with a tuple containing the input and return values.

Symmetric key generation queries: Let $I D_{s}, I D_{r}$ be the identity of the sender and that of the receiver respectively used by $\mathcal{A}$ in a symmetric key generation query. For each new query $\left(I D_{s}, I D_{r}\right)$, $\mathcal{C}$ proceeds as follows:

1. If $I D_{s} \neq I D_{j}, \mathcal{C}$ computes the private key $S_{I D_{s}}$ corresponding to $I D_{s}$ by running the private key extraction query algorithm. Then $\mathcal{C}$ runs $(K, \omega) \leftarrow \operatorname{Sym}\left(\right.$ params, $\left.S_{I D_{s}}, I D_{s}, P K_{I D_{s}}, I D_{r}, P K_{I D_{r}}\right)$ and sends $K$ to $\mathcal{A}$. Note that $\mathcal{C}$ needs to store $\omega$ and to overwrite any previous value.

2. If $I D_{s}=I D_{j}$ (and hence $I D_{r} \neq I D_{j}$ ), $\mathcal{C}$ chooses $u, v \in Z_{q}^{*}$, sets $U \leftarrow v a P$, and computes $T \leftarrow \hat{e}\left(U, D_{I D_{r}}\right)\left(\mathcal{C}\right.$ could obtain $D_{I D_{r}}$ from a partial private key extraction query because $\left.I D_{r} \neq I D_{j}\right)$. Note that the $\omega$ is $\left(u, v, U, I D_{s}, P K_{I D_{s}}, I D_{r}, P K_{I D_{r}}\right)$ in this case.

3. It goes through list $L_{2}$ looking for an entry $\left(U, T, R, I D_{r}, P K_{I D_{r}}, h\right)$ for some $R$ such that $\hat{e}\left(U, P K_{I D_{r}}\right)=\hat{e}(P, R)$, where $P K_{I D_{r}}$ is obtained by calling the request public key oracle on $I D_{r}$. If such an entry exists, it computes $K \leftarrow h$. Otherwise it uses a random $h$ and updates the list $L_{2}$ with $\left(U, T, \star, I D_{r}, P K_{I D_{r}}, h\right)$.

Key encapsulation queries: $\mathcal{A}$ produces a arbitrary tag $\tau$. $\mathcal{C}$ checks whether there exists a stored value $\omega$. If there is not, it returns $\perp$ and terminates. Otherwise $\mathcal{C}$ proceeds as follows.

1. If $I D_{s} \neq I D_{j}, \mathcal{C}$ answers the query by a call to $\operatorname{Encap}(\omega, \tau)$.

2. If $I D_{s}=I D_{j}$ (and $\left.I D_{r} \neq I D_{j}\right), \mathcal{C}$ defines the hash value $H_{3}\left(U, \tau, I D_{s}, P K_{I D_{s}}\right)$ as $H \leftarrow$ $v^{-1}\left(u P-Q_{I D_{s}}\right)$. If a such a hash queries has been responded with a different value before, it aborts the simulation. This means that $\mathcal{C}$ updates list $L_{3}$ with tuple $\left(U, \tau, I D_{s}, P K_{I D_{s}}, \perp, H\right)$. Finally, $\mathcal{C}$ sets $W=u a P+l P K_{I D_{s}}$, where $l$ is the value obtained by querying $H_{4}$ on $\left(U, \tau, I D_{s}, P K_{I D_{s}}\right)$. $\mathcal{C}$ returns $\psi \leftarrow(U, W)$.

Key decapsulation queries: For a key decapsulation query on a $\left(\psi^{\prime}, \tau^{\prime}\right)$ for identities $I D_{s}$ and $I D_{r}, \mathcal{C}$ proceeds as follows.

1. It executes the verification part of the decapsulation algorithm by obtaining $Q_{I D_{s}}$ and $P K_{I D_{s}}$ by calling $H_{1}$ and request public key oracles. It returns $\perp$ if the verification does not succeed.

2. It computes $R \leftarrow x_{I D_{r}} U$, obtaining $x_{I D_{r}}$ (and hence $P K_{I D_{r}}$ ) from either the adversary or by calling the request public key oracle.

3. If $I D_{r} \neq I D_{j}, \mathcal{C}$ computes the partial private key $D_{I D_{r}}$ corresponding to $I D_{r}$ by running the partial private key extraction query algorithm. Then $\mathcal{C}$ computes $T \leftarrow \hat{e}\left(D_{I D_{r}}, U\right)$, and returns $K \leftarrow H_{2}\left(U, T, R, I D_{r}, P K_{I D_{r}}\right)$.

4. If $I D_{r}=I D_{j}$, then the pairing cannot be computed. In order to return a consistent answer, $\mathcal{C}$ goes through $L_{2}$ and looks for a tuple $\left(U, T, R, I D_{r}, P K_{I D_{r}}, h\right)$, for different values of $T$, such that the decision bilinear Diffie-Hellman oracle returns 1 when queried on $(a P, b P, U, T)$. If such an entry exists, the correct pairing value is found and returns $K \leftarrow h$.

5. If $\mathcal{C}$ reaches this point of execution, it places the entry $\left(U, \star, R, I D_{r}, P K_{I D_{r}}, h\right)$ for a random $h$ on list $L_{2}$ and returns $K \leftarrow h$. The symbol $\star$ denotes an unknown value of pairing. Note that the identity component of all entries with a $\star$ is $I D_{j}$. 
After the first stage, $\mathcal{A}$ picks two identities $I D_{s}^{*}$ and $I D_{r}^{*}$ on which it wishes to be challenged. If $I D_{r}^{*} \neq I D_{j}, \mathcal{C}$ fails and stops. Otherwise it proceeds to construct a challenge as follows. It obtains the public key $P K_{I D_{s}^{*}}$ corresponding to $I D_{s}^{*}$ form the list $L_{K}$. Then it sets $U^{*}=c P$, chooses a random hash value $h^{*}$ and sets $K_{1} \leftarrow h^{*}$. $\mathcal{C}$ chooses $K_{0} \leftarrow \mathcal{K}_{\text {CLSC-TKEM }}$ and a bit $b \in\{0,1\}$ randomly, and sends $K_{b}$ to $\mathcal{A}$. $\mathcal{A}$ then sends a tag $\tau^{*}$ to $\mathcal{C}$. $\mathcal{C}$ computes $W^{*}=D_{I D_{s}^{*}}+r H+x_{I D_{s}^{*}} H^{\prime}=$ $D_{I D_{s}^{*}}+t c P+l P K_{I D_{s}^{*}}$, where $t$ is obtained from $L_{3}, l$ is obtained from $L_{4}$ and $D_{I D_{s}^{*}}$ is computed by calling the partial private key extraction oracle on $I D_{s}^{*}$. Note that, since $I D_{s}^{*} \neq I D_{r}^{*}$ the partial private key extraction oracle simulation always give $\mathcal{C}$ the correct value of $D_{I D_{s}}$. $\mathcal{C}$ sends the challenge encapsulation $\psi^{*} \leftarrow\left(U^{*}, W^{*}\right)$ to $\mathcal{A}$.

$\mathcal{A}$ then performs a second series of queries which is treated in the same way as the first one. At the end of the simulation, it produces a bit $b^{\prime}$ for which it believes the relation $\left(K_{b}, \omega^{*}\right) \leftarrow$ $\operatorname{Sym}\left(\right.$ params, $\left.S_{I D_{s}^{*}}, I D_{s}^{*}, P K_{I D_{s}^{*}}, I D_{r}^{*}, P K_{I D_{r}^{*}}\right)$ and $\psi^{*} \leftarrow \operatorname{Encap}\left(\omega^{*}, \tau^{*}\right)$ hold.

Since $I D_{j}$ is independent of adversary's view, and the list $L_{1}$ can be easily seen to have at most $q_{T}$ elements, with probability $1 / q_{T}$ the adversary will output an identity $I D_{j}$. If this event occurs, the simulation is perfect unless the adversary queries $H_{2}$ on the challenge-related tuple $\left(U^{*}, T^{*}, R^{*}, I D_{r}^{*}, P K_{I D_{r}}^{*}\right)$. Since the hash function $H_{2}$ is modeled as a random oracle, the adversary will not have any advantage if this tuple does not appear on $L_{2}$. However, if this happens, $\mathcal{C}$ will win the game due to the first step in the simulation of $\mathrm{H}_{2}$. The Lemma follows from this observation and the fact that the total number of decision bilinear Diffie-Hellman oracle calls that $\mathcal{C}$ makes is at most $q_{D}^{2}+2 q_{D} q_{2}+q_{2}$.

Lemma 6. Under the $C D H$ assumption in $G_{1}$, no PPT attacker $\mathcal{A}$ has non-negligible advantage in winning the IND-CCA-II game against the above CLSC-TKEM, when all hash functions are modeled as random oracles. More precisely, there exists an algorithm $\mathcal{C}$ which uses $\mathcal{A}$ to solve the CDH problem such that:

$$
\operatorname{Adv}_{\mathrm{CLSC}}^{\mathrm{IND}-\mathrm{CCA} 2-\mathrm{II}}(\mathcal{A}) \leq q_{T} \operatorname{Adv}_{C D H}(\mathcal{C})
$$

where $q_{T}=q_{R K}+q_{P K}+q_{K}+2 q_{D}+2$. Here $q_{R K}$ and $q_{P K}$ are the maximum number of queries that the adversary can request public key and replace public key, respectively. $q_{K}$ and $q_{D}$ are as before.

Proof. The challenger $\mathcal{C}$ takes as input $(P, a P, b P)$ and attempts to compute $a b P . \mathcal{C}$ will run $\mathcal{A}$ as a subroutine and act as $\mathcal{A}$ 's challenger in the IND-CCA2-II game for CLSC-TKEM. During the game, $\mathcal{A}$ will consult $\mathcal{C}$ for answers to the random oracles $H_{1}, H_{2}, H_{3}$ and $H_{4}$. Roughly speaking, these answers are randomly generated, but to maintain the consistency and to avoid collision, $\mathcal{C}$ keeps three lists $L_{1}, L_{2}, L_{3}, L_{4}$ respectively to store the answers. The following assumptions are made.

1. $\mathcal{A}$ will ask for $H_{1}(I D)$ before $I D$ is used in any private key extraction, symmetric key generation, key encapsulation and key decapsulation queries.

2. Key encapsulation returned from a key encapsulation query will not be used by $\mathcal{A}$ in a key decapsulation query.

At the beginning of the game, $\mathcal{C}$ generates a master secret key $s$ and system parameters params including a master public key $P_{p u b} \leftarrow s P$. Then $\mathcal{C}$ gives both params and $s$ to $\mathcal{A}$. $\mathcal{C}$ first chooses a random number $j \in\left\{1,2, \ldots, q_{T}\right\}$, and answers various oracle queries as follows. 
$H_{1}$ queries: For a query on $H_{1}\left(I D_{i}\right), \mathcal{C}$ chooses $e_{i} \in Z_{q}^{*}$ randomly, puts $\left(I D_{i}, e_{i}\right)$ in list $L_{1}$ and answers $H_{1}\left(I D_{i}\right) \leftarrow e_{i} P$.

Request public key: When $\mathcal{A}$ asks a public key query on identity $I D_{i}$, if $I D_{i} \neq I D_{j}, \mathcal{C}$ generates a new key pair $\left(x_{I D_{i}}, P K_{I D_{i}}\right)$, updates the list $L_{K}$ with $\left(I D_{i}, P K_{I D_{i}}, x_{I D_{i}}\right)$, and returns the public key. If $I D_{i}=I D_{j}, \mathcal{C}$ returns $a P$ and adds $\left(I D_{j}, a P, \perp\right)$ to $L_{K}$.

Extract private key: When $\mathcal{A}$ asks a private key extraction query on identity $I D_{i}, \mathcal{C}$ calls request public key on $I D_{i}$ and obtains $\left(I D_{i}, P K_{I D_{i}}, x_{I D_{i}}\right)$. If $I D_{i}=I D_{j}$, then $\mathcal{C}$ fails and stops. Otherwise, $\mathcal{C}$ calls $H_{1}$ on $I D_{i}$ and gets $\left(I D_{i}, e_{i}\right)$. It returns $\left(x_{I D_{i}}, s e_{i} P\right)$.

$H_{3}$ Queries: When $\mathcal{A}$ asks a $H_{3}$ query on $\left(U_{i}, \tau_{i}, I D_{i}, P K_{I D_{i}}\right), \mathcal{C}$ checks if the list $L_{3}$ contains a tuple $\left(U_{i}, \tau_{i}, I D_{i}, P K_{I D_{i}}, t_{i}, t_{i} P\right)$. If such a tuple is found, $\mathcal{C}$ answers $t_{i} P$. Otherwise, $\mathcal{C}$ chooses a random value $t \in Z_{q}$, puts the $\left(U_{i}, \tau_{i}, I D_{i}, P K_{I D_{i}}, t, t P\right)$ into $L_{3}$, and returns $t P$.

$H_{4}$ Queries: When $\mathcal{A}$ asks a $H_{4}$ query on $\left(U_{i}, \tau_{i}, I D_{i}, P K_{I D_{i}}\right), \mathcal{C}$ checks if the list $L_{4}$ contains a tuple $\left(U_{i}, \tau_{i}, I D_{i}, P K_{I D_{i}}, l_{i}, l_{i} P\right)$. If such a tuple is found, $\mathcal{C}$ answers $l_{i} P$. Otherwise, $\mathcal{C}$ chooses a random value $l \in Z_{q}$, puts the $\left(U_{i}, \tau_{i}, I D_{i}, P K_{I D_{i}}, l, l P\right)$ into $L_{4}$, and returns $l P$.

$H_{2}$ queries: For each new query $\left(U_{i}, T_{i}, R_{i}, I D_{i}, P K_{I D_{i}}\right), \mathcal{C}$ proceeds as follows:

1. It checks if $\hat{e}(a P, b P)=\hat{e}\left(P, R_{i}\right)$. If so, $\mathcal{C}$ returns $R_{i}$ and stops.

2. $\mathcal{C}$ goes through the list $L_{2}$ looking for entries $\left(U_{i}, T_{i}, \star, I D_{i}, P K_{I D_{i}}, h_{i}\right)$ such that $\hat{e}\left(U_{i}, a P\right)=$ $\hat{e}\left(P, R_{i}\right)$. Note that in this case $I D_{i}=I D_{j}$. If such a tuple exists, it returns $h_{i}$ (and replaces the symbol $\star$ with $R_{i}$ ).

3. If $\mathcal{C}$ reaches this point of execution, it returns a random $h$ and updates the list $L_{2}$, which is initially empty, with a tuple containing the input and return values.

Symmetric key generation queries: Let $I D_{s}, I D_{r}$ be the identity of the sender and that of the receiver respectively used by $\mathcal{A}$ in a symmetric key generation query. For each new query $\left(I D_{s}, I D_{r}\right)$, $\mathcal{C}$ proceeds as follows:

1. If $I D_{s} \neq I D_{j}, \mathcal{C}$ computes the private key $S_{I D_{s}}$ corresponding to $I D_{s}$ by running the private key extraction query algorithm. Then $\mathcal{C}$ runs $(K, \omega) \leftarrow \operatorname{Sym}\left(\right.$ params, $\left.S_{I D_{s}}, I D_{s}, P K_{I D_{s}}, I D_{r}, P K_{I D_{r}}\right)$ and sends $K$ to $\mathcal{A}$. Note that $\mathcal{C}$ needs to store $\omega$ and to overwrite any previous value.

2. If $I D_{s}=I D_{j}$ (and hence $\left.I D_{r} \neq I D_{j}\right), \mathcal{C}$ chooses $u, v \in Z_{q}^{*}$, sets $U \leftarrow v a P$, and computes $T \leftarrow \hat{e}\left(U, D_{I D_{r}}\right)\left(\mathcal{C}\right.$ could computes $D_{I D_{r}}$ because it knows the master secret key $\left.s\right)$. Note that the $\omega$ is $\left(u, v, U, I D_{s}, P K_{I D_{s}}, I D_{r}, P K_{I D_{r}}\right)$ in this case.

3. It goes through list $L_{2}$ looking for an entry $\left(U, T, R, I D_{r}, P K_{I D_{r}}, h\right)$ for some $R$ such that $\hat{e}\left(U, P K_{I D_{r}}\right)=\hat{e}(P, R)$, where $P K_{I D_{r}}$ is obtained by calling the request public key oracle on $I D_{r}$. If such an entry exists, it computes $K \leftarrow h$. Otherwise it uses a random $h$ and updates the list $L_{2}$ with $\left(U, T, \star, I D_{r}, P K_{I D_{r}}, h\right)$.

Key encapsulation queries: $\mathcal{A}$ produces a arbitrary tag $\tau$. $\mathcal{C}$ checks whether there exists a stored value $\omega$. If there is not, it returns $\perp$ and terminates. Otherwise $\mathcal{C}$ proceeds as follows.

1. If $I D_{s} \neq I D_{j}, \mathcal{C}$ answers the query by a call to $\operatorname{Encap}(\omega, \tau)$.

2. If $I D_{s}=I D_{j}\left(\right.$ and $\left.I D_{r} \neq I D_{j}\right), \mathcal{C}$ defines the hash value $H_{3}\left(U, \tau, I D_{s}, P K_{I D_{s}}\right)$ as $H \leftarrow$ $v^{-1}\left(u P-H_{4}\right)$, where $H_{4}$ is the output of $H_{4}\left(U, \tau, I D_{s}, P K_{I D_{s}}\right)$. If a such a hash queries has been responded with a different value before, it aborts the simulation. This means that $\mathcal{C}$ updates list $L_{3}$ with tuple $\left(U, \tau, I D_{s}, P K_{I D_{s}}, \perp, H\right)$. Finally, $\mathcal{C}$ sets $W=D_{I D_{s}}+$ uaP. $\mathcal{C}$ returns $\psi \leftarrow(U, W)$. 
Key decapsulation queries: For a key decapsulation query on a $\left(\psi^{\prime}, \tau^{\prime}\right)$ for identities $I D_{s}$ and $I D_{r}, \mathcal{C}$ proceeds as follows.

1. It executes the verification part of the decapsulation algorithm obtaining $Q_{I D_{s}}$ and $P K_{I D_{s}}$ by calling $H_{1}$ and request public key oracles. It returns $\perp$ if the verification does not succeed.

2. It calculates $T=\hat{e}\left(U, e_{r} P_{p u b}\right)$, where $\left(I D_{r}, e_{r}\right)$ is obtained from $H_{1}$.

3. If $I D_{r} \neq I D_{j}$, it computes $R \leftarrow x_{I D_{r}} U$, where $x_{I D_{r}}$ is obtained (and hence $P K_{I D_{r}}$ ) from either the adversary or by calling the request public key oracle. Then $\mathcal{C}$ returns $K \leftarrow H_{2}\left(U, T, R, I D_{r}, P K_{I D_{r}}\right)$.

4. If $I D_{r}=I D_{j}$, the correct value of $R$ cannot be computed. To return a consistent answer, $\mathcal{C}$ goes through $L_{2}$ and looks for a tuple $\left(U, T, R, I D_{r}, P K_{I D_{r}}, h\right)$, for different values of $R$, such that $\hat{e}(U, a P)=\hat{e}(P, R)$. If such an entry exists, the correct value of $R$ is found and returns $K \leftarrow h$.

5. If $\mathcal{C}$ reaches this point of execution, it places the entry $\left(U, T, \star, I D_{r}, P K_{I D_{r}}, h\right)$ for a random $h$ on list $L_{2}$ and returns $K \leftarrow h$. The symbol $\star$ denotes an unknown value of $R$.

After the first stage, $\mathcal{A}$ picks two identities $I D_{s}^{*}$ and $I D_{r}^{*}$ on which it wishes to be challenged. If $I D_{r}^{*} \neq I D_{j}, \mathcal{C}$ fails and stops. Otherwise it proceeds to construct a challenge as follows. It obtains the public key $P K_{I D_{s}^{*}}$ corresponding to $I D_{s}^{*}$ form the list $L_{K}$. Then it sets $U^{*}=b P$, chooses a random hash value $h^{*}$ and sets $K_{1} \leftarrow h^{*}$. $\mathcal{C}$ chooses $K_{0} \leftarrow \mathcal{K}_{\text {CLSC-TKEM }}$ and a bit $b \in\{0,1\}$ randomly, and sends $K_{b}$ to $\mathcal{A}$. $\mathcal{A}$ then sends a tag $\tau^{*}$ to $\mathcal{C}$. $\mathcal{C}$ computes $W^{*}=D_{I D_{s}^{*}}+r H+x_{I D_{s}^{*}} H^{\prime}=$ $D_{I D_{s}^{*}}+t c P+l P K_{I D_{s}^{*}}$, where $t$ is obtained from $L_{3}, l$ is obtained from $L_{4}$ and $D_{I D_{s}^{*}}$ is computed by calling the partial private key extraction oracle on $I D_{s}^{*} \cdot \mathcal{C}$ sends the challenge encapsulation $\psi^{*} \leftarrow\left(U^{*}, W^{*}\right)$ to $\mathcal{A}$.

$\mathcal{A}$ then performs a second series of queries which is treated in the same way as the first one. At the end of the simulation, it produces a bit $b^{\prime}$ for which it believes the relation $\left(K_{b}, \omega^{*}\right) \leftarrow$ $\operatorname{Sym}\left(\right.$ params $\left., S_{I D_{s}^{*}}, I D_{s}^{*}, P K_{I D_{s}^{*}}, I D_{r}^{*}, P K_{I D_{r}^{*}}\right)$ and $\psi^{*} \leftarrow \operatorname{Encap}\left(\omega^{*}, \tau^{*}\right)$ hold.

Since $I D_{j}$ is independent of adversary's view, and the list $L_{1}$ can be easily seen to have at most $q_{T}$ elements, with probability $1 / q_{T}$ the adversary will output an identity $I D_{j}$. If this event occurs, the simulation is perfect unless the adversary queries $H_{2}$ on the challenge-related tuple $\left(U^{*}, T^{*}, R^{*}, I D_{r}^{*}, P K_{I D_{r}}^{*}\right)$. Since the hash function $H_{2}$ is modeled as a random oracle, the adversary will not have any advantage if this tuple does not appear on $L_{2}$. However, if this happens, $\mathcal{C}$ will win the game due to its simulation of $H_{2}$. The Lemma follows from this observation and the fact that the maximum length of the list $L_{K}$ is $q_{T}$, as stated in the Lemma.

\section{E Proof of Theorem 4}

Proof. In the Barbosa-Farshim CLSC scheme [6], they use a weaker formulation of Type I adversary which they refer to as Type $\mathrm{I}^{\prime}$. In unforgeability games, the Type $\mathrm{I}^{\prime}$ adversary is not allowed to extract the partial private key of $I D_{s}^{*}$. They proved that If a CLSC scheme is sUF-CMA secure against Type II and Type $\mathrm{I}^{\prime}$ attackers, then it is also sUF-CMA secure against Type I attackers. It is easy to extend these conclusions to CLSC-TKEM setting. That is, we have the following Lemma 7.

Lemma 7. If a CLSC-TKEM is sUF-CMA secure against Type II and Type I' attackers then it is also sUF-CMA secure against Type I attackers. In particular, we have

$$
\operatorname{Adv}_{\mathrm{CLSC}}^{\mathrm{sUF}-\mathrm{CMA}-\mathrm{I}}(\mathcal{F}) \leq 2 \operatorname{Adv}_{\mathrm{CLSC}}^{\mathrm{sUF}-\mathrm{CMA}-\mathrm{I}^{\prime}}\left(\mathcal{C}_{1}\right)+\operatorname{Adv}_{\mathrm{CLSC}}^{\mathrm{sUF}-\mathrm{CMA}-\mathrm{II}}\left(\mathcal{C}_{2}\right)
$$


This theorem follows from Lemmas 7, 8 and 9 .

Lemma 8. Under the $G D H^{\prime}$ assumption in $G_{1}$, no PPT attacker $\mathcal{F}$ has non-negligible advantage in winning the sUF-CMA-I' game against the above CLSC-TKEM, when all hash functions are modeled as random oracles. More precisely, there exists an algorithm $\mathcal{C}$ which uses $\mathcal{F}$ to solve the GDH' problem such that:

$$
\operatorname{Adv}_{\mathrm{CLSC}}^{\mathrm{sUF}-\mathrm{CMA}^{\prime} \mathrm{I}^{\prime}}(\mathcal{F}) \leq q_{T} \operatorname{Adv}_{G D H^{\prime}}\left(\mathcal{C}, q_{D}^{2}+2 q_{D} q_{2}\right)+\left(q_{S K}\left(q_{S K}+q_{D}+q_{3}+1\right)+2\right) / 2^{k},
$$

where $q_{T}=q_{1}+q_{P}+q_{K}+2 q_{D}+2 q_{S K}+1$. Here $q_{3}$ and $q_{S K}$ are the maximum number of queries that the adversary could ask $H_{3}$ and make symmetric key generation queries, respectively. $q_{1}, q_{P}$, $q_{K}$, and $q_{D}$ are as before.

Proof. The challenger $\mathcal{C}$ takes as input $(P, a P, b P)$ and attempts to compute $a b P . \mathcal{C}$ will run $\mathcal{A}$ as a subroutine and act as $\mathcal{A}$ 's challenger in the sUF-CMA-I' game for CLSC-TKEM. During the game, $\mathcal{A}$ will consult $\mathcal{C}$ for answers to the random oracles $H_{1}, H_{2}, H_{3}$ and $H_{4}$. Roughly speaking, these answers are randomly generated, but to maintain the consistency and to avoid collision, $\mathcal{C}$ keeps three lists $L_{1}, L_{2}, L_{3}, L_{4}$ respectively to store the answers. The following assumptions are made.

1. $\mathcal{F}$ will ask for $H_{1}(I D)$ before $I D$ is used in any partial private key extraction, private key extraction, symmetric key generation, key encapsulation and key decapsulation queries.

2. Key encapsulation returned from a key encapsulation query will not be used by $\mathcal{F}$ in a key decapsulation query.

At the beginning of the game, $\mathcal{C}$ gives $\mathcal{F}$ the system parameters with $P_{\text {pub }} \leftarrow a P$. Note that $a$ is unknown to $\mathcal{C}$. This value simulates the master key value for the $\mathrm{KGC}$ in the game. $\mathcal{C}$ chooses a random number $j \in\left\{1,2, \ldots, q_{T}\right\}$ and answers various oracle queries as follows.

$H_{1}$ queries: Same to Lemma 5.

Extract partial private key: Same to Lemma 5.

Request public key: Same to Lemma 5.

Replace public key: Same to Lemma 5.

Extract private key: Same to Lemma 5.

$\mathrm{H}_{3}$ Queries: Same to Lemma 5.

$H_{4}$ Queries: Same to Lemma 5.

$H_{2}$ queries: For each new query $\left(U_{i}, T_{i}, R_{i}, I D_{i}, P K_{I D_{i}}\right), \mathcal{C}$ proceeds as follows:

1. It checks if $\hat{e}(a P, b P)=\hat{e}\left(P, R_{i}\right)$. If this is the case, $\mathcal{C}$ returns $R_{i}$ and stop.

2. $\mathcal{C}$ goes through the list $L_{2}$ with entries $\left(U_{i}, \star, R_{i}, I D_{i}, P K_{I D_{i}}, h_{i}\right)$, for different values of $h_{i}$, such that the decision bilinear Diffie-Hellman oracle returns 1 when queried on the tuple $\left(a P, b P, U_{i}, T_{i}\right)$. Note that in this case $I D_{i}=I D_{j}$. If such a tuple exists, it returns $h_{i}$ (and replaces the symbol $\star$ with $T_{i}$ )

3. It goes through the list $L_{2}$ with entries $\left(U_{i}, T_{i}, \star, I D_{i}, P K_{I D_{i}}, h_{i}\right)$, for different values of $h_{i}$, such that $\hat{e}\left(U_{i}, P K_{I D_{i}}\right)=\hat{e}\left(P, R_{i}\right)$. If such a tuple exists, it returns $h_{i}$ (and replaces the symbol $\star$ with $R_{i}$ ). 
4. If $\mathcal{C}$ reaches this point of execution, it returns a random $h$ and updates the list $L_{2}$, which is initially empty, with a tuple containing the input and return values.

Symmetric key generation queries: Let $I D_{s}, I D_{r}$ be the identity of the sender and that of the receiver respectively used by $\mathcal{F}$ in a symmetric key generation query. For each new query $\left(I D_{s}, I D_{r}\right)$, $\mathcal{C}$ proceeds as follows:

1. If $I D_{s} \neq I D_{j}, \mathcal{C}$ computes the private key $S_{I D_{s}}$ corresponding to $I D_{s}$ by running the private key extraction query algorithm. Then $\mathcal{C}$ runs $(K, \omega) \leftarrow \operatorname{Sym}\left(\right.$ params, $\left.S_{I D_{s}}, I D_{s}, P K_{I D_{s}}, I D_{r}, P K_{I D_{r}}\right)$ and sends $K$ to $\mathcal{F}$. Note that $\mathcal{C}$ needs to store $\omega$ and to overwrite any previous value.

2. If $I D_{s}=I D_{j}$ (and hence $I D_{r} \neq I D_{j}$ ), $\mathcal{C}$ chooses $u, v \in Z_{q}^{*}$, sets $U \leftarrow v a P$, and computes $T \leftarrow \hat{e}\left(U, D_{I D_{r}}\right)\left(\mathcal{C}\right.$ could obtain $D_{I D_{r}}$ from a partial private key extraction query because $\left.I D_{r} \neq I D_{j}\right)$. Note that the $\omega$ is $\left(u, v, U, I D_{s}, P K_{I D_{s}}, I D_{r}, P K_{I D_{r}}\right)$ in this case.

3. It goes through list $L_{2}$ looking for an entry $\left(U, T, R, I D_{r}, P K_{I D_{r}}, h\right)$ for some $R$ such that $\hat{e}\left(U, P K_{I D_{r}}\right)=\hat{e}(P, R)$, where $P K_{I D_{r}}$ is obtained by calling the request public key oracle on $I D_{r}$. If such an entry exists, it computes $K \leftarrow h$. Otherwise it uses a random $h$ and updates the list $L_{2}$ with $\left(U, T, \star, I D_{r}, P K_{I D_{r}}, h\right)$.

Key encapsulation queries: $\mathcal{F}$ produces a arbitrary tag $\tau$. $\mathcal{C}$ checks whether there exists a stored value $\omega$. If there is not, it returns $\perp$ and terminates. Otherwise $\mathcal{C}$ proceeds as follows.

1. If $I D_{s} \neq I D_{j}, \mathcal{C}$ answers the query by a call to $\operatorname{Encap}(\omega, \tau)$.

2. If $I D_{s}=I D_{j}$ (and $\left.I D_{r} \neq I D_{j}\right), \mathcal{C}$ defines the hash value $H_{3}\left(U, \tau, I D_{s}, P K_{I D_{s}}\right)$ as $H \leftarrow$ $v^{-1}\left(u P-Q_{I D_{s}}\right)$. If a such a hash queries has been responded with a different value before, it aborts the simulation. This means that $\mathcal{C}$ updates list $L_{3}$ with tuple $\left(U, \tau, I D_{s}, P K_{I D_{s}}, \perp, H\right)$. Finally, $\mathcal{C}$ sets $W=u a P+l P K_{I D_{s}}$, where $l$ is the value obtained by querying $H_{4}$ on $\left(U, \tau, I D_{s}, P K_{I D_{s}}\right)$. $\mathcal{C}$ returns $\psi \leftarrow(U, W)$.

Key decapsulation queries: For a key decapsulation query on a $\left(\psi^{\prime}, \tau^{\prime}\right)$ for identities $I D_{s}$ and $I D_{r}, \mathcal{C}$ proceeds as follows.

1. It executes the verification part of the decapsulation algorithm obtaining $Q_{I D_{s}}$ and $P K_{I D_{s}}$ by calling $H_{1}$ and request public key oracles. It returns $\perp$ if the verification does not succeed.

2. It checks if $I D_{i}=I D_{j}$ and if this is the case then $\mathcal{C}$ can solve the $\mathrm{GDH}^{\prime}$ problem as described below.

3. It computes $R \leftarrow x_{I D_{r}} U$, obtaining $x_{I D_{r}}$ (and hence $P K_{I D_{r}}$ ) from either the adversary or by calling the request public key oracle.

4. If $I D_{r} \neq I D_{j}, \mathcal{C}$ computes the private key $D_{I D_{r}}$ corresponding to $I D_{r}$ by running the partial private key extraction query algorithm. Then $\mathcal{C}$ computes $T \leftarrow \hat{e}\left(D_{I D_{r}}, U\right)$, and returns $K \leftarrow$ $H_{2}\left(U, T, R, I D_{r}, P K_{I D_{r}}\right)$.

5. If $I D_{r}=I D_{j}$, then the pairing cannot be computed. In order to return a consistent answer, $\mathcal{C}$ goes through $L_{2}$ and looks for a tuple $\left(U, T, R, I D_{r}, P K_{I D_{r}}, h\right)$, for different values of $T$, such that the decision bilinear Diffie-Hellman oracle returns 1 when queried on $(a P, b P, U, T)$. If such an entry exists, the correct pairing value is found and returns $K \leftarrow h$.

6. If $\mathcal{C}$ reaches this point of execution, it places the entry $\left(U, \star, R, I D_{r}, P K_{I D_{r}}, h\right)$ for a random $h$ on list $L_{2}$ and returns $K \leftarrow h$. The symbol $\star$ denotes an unknown value of pairing. Note that the identity component of all entries with a $\star$ is $I D_{j}$. 
Finally, $\mathcal{F}$ outputs a produces a quaternion $\left(\tau^{*}, \psi^{*}, I D_{s}^{*}, I D_{r}^{*}\right) \cdot \mathcal{C}$ checks if $I D_{s}^{*}=I D_{j}$. If not, it aborts execution. Otherwise, it obtains $P K_{I D_{s}^{*}}$ by calling the request public key oracle on $I D_{s}^{*}$ and retrieves $t^{*}$ and $l^{*}$ from lists $L_{3}$ and $L_{4}$ by querying $H_{3}$ and $H_{4}$ on $\left(U^{*}, \tau^{*}, I D_{s}^{*}, P K_{I D_{s}^{*}}\right)$. Note that if $\mathcal{C}$ succeeded, then the verification condition holds:

$$
\begin{aligned}
\hat{e}\left(P, W^{*}\right) & =\hat{e}\left(P_{p u b}, Q_{I D_{s}^{*}}\right) \hat{e}\left(U^{*}, H^{*}\right) \hat{e}\left(P K_{I D_{s}^{*}}, H^{* *}\right) \\
\hat{e}\left(P, W^{*}\right) & =\hat{e}(a P, b P) \hat{e}\left(U^{*}, t^{*} P\right) \hat{e}\left(P K_{I D_{s}^{*}}, l^{*} P\right) \\
\hat{e}(P, a b P) & =\hat{e}\left(P, W^{*}-t^{*} U-l^{*} P K_{I D_{s}^{*}}\right)
\end{aligned}
$$

and thus $\mathcal{C}$ can compute

$$
a b P=W^{*}-t^{*} U-l^{*} P K_{I D_{s}^{*}}
$$

Let us now analyze the probability that $\mathcal{C}$ succeeds in solving the $\mathrm{GDH}^{\prime}$ problem instance. For this to happen, the simulation must run until the end of the game, the adversary must pick a specific identity as $I D_{j}^{*}$, and it must query the hash functions $H_{3}$ and $H_{4}$ to properly construct the forgery. The probability that $\mathcal{F}$ is able to produce a forgery without querying both hash functions is upper bounded by $2 / 2^{k}$.

The probability that $\mathcal{C}$ aborts the simulation is related with the following events:

$-\mathcal{F}$ places a partial key extraction on $I D_{j}$.

- $\mathcal{F}$ places a full private key extraction on $I D_{j}$.

- $\mathcal{C}$ wants to simulate a key encapsulation query and this leads to an inconsistency in the $H_{3}$ simulation.

Note that if $\mathcal{F}$ places either of the first two fatal queries, then it could not possibly use $I D_{j}$ as the sender identity in the forgery it produces at the end of the game, so we can pinpoint the probability that $\mathcal{C}$ does not abort the simulation due to these events and $\mathcal{F}$ picks the only useful case for solving $\mathrm{GDH}^{\prime}$ as $1 / q_{T}$. Note that the maximum length of the list $L_{1}$ is $q_{T}$, as stated in the Lemma

The latter fatal event occurs if $\mathcal{C}$ 's simulation triggers a collision in its simulation of $H_{3}$. Since the maximum size of $L_{3}$ is $q_{S K}+q_{D}+q_{3}+1$, we can upper bound the probability that this occurs as $q_{S K}\left(q_{S K}+q_{D}+q_{3}+1\right) / 2^{k}$. The result follows by noting that $\mathcal{C}$ makes at most $q_{D}^{2}+2 q_{D} q_{2}$ queries to its decision bilinear Diffie-Hellman oracle.

Lemma 9. Under the $C D H$ assumption in $G_{1}$, no PPT attacker $\mathcal{F}$ has non-negligible advantage in winning the sUF-CMA-II game against the above CLSC-TKEM, when all hash functions are modeled as random oracles. More precisely, there exists an algorithm $\mathcal{C}$ which uses $\mathcal{F}$ to solve the CDH problem such that:

$$
\operatorname{Adv}_{\mathrm{CLSC}}^{\mathrm{sUF}-\mathrm{CMA}-\mathrm{II}}(\mathcal{F}) \leq q_{T} \operatorname{Adv}_{C D H}(\mathcal{C})+\left(q_{S K}\left(q_{S K}+q_{D}+q_{3}+1\right)+2\right) / 2^{k},
$$

where $q_{T}=q_{R K}+q_{P K}+q_{K}+2 q_{D}+2 q_{S K}+1$ and various $q$ 's are as before.

Proof. The challenger $\mathcal{C}$ takes as input $(P, a P, b P)$ and attempts to compute $a b P . \mathcal{C}$ will run $\mathcal{F}$ as a subroutine and act as $\mathcal{F}$ 's challenger in the sUF-CMA-II game for CLSC-TKEM. During the game, $\mathcal{F}$ will consult $\mathcal{C}$ for answers to the random oracles $H_{1}, H_{2}, H_{3}$ and $H_{4}$. Roughly speaking, these answers are randomly generated, but to maintain the consistency and to avoid collision, $\mathcal{C}$ keeps three lists $L_{1}, L_{2}, L_{3}, L_{4}$ respectively to store the answers. The following assumptions are made. 
1. $\mathcal{F}$ will ask for $H_{1}(I D)$ before $I D$ is used in any private key extraction, symmetric key generation, key encapsulation and key decapsulation queries.

2. Key encapsulation returned from a key encapsulation query will not be used by $\mathcal{F}$ in a key decapsulation query.

At the beginning of the game, $\mathcal{C}$ generates a master secret key $s$ and system parameters params including a master public key $P_{p u b}$. Then $\mathcal{C}$ gives both params and $s$ to $\mathcal{F}$. $\mathcal{C}$ first chooses a random number $j \in\left\{1,2, \ldots, q_{T}\right\}$, and answers various oracle queries as follows.

$H_{1}$ queries: Same to Lemma 6.

Request public key: Same to Lemma 5.

Extract private key: Same to Lemma 5.

$H_{3}$ Queries: Same to Lemma 6.

$H_{4}$ Queries: When $\mathcal{F}$ asks a $H_{4}$ query on $\left(U_{i}, V_{i}, I D_{i}, P K_{I D_{i}}\right), \mathcal{C}$ checks if the list $L_{4}$ contains a tuple $\left(U_{i}, V_{i}, I D_{i}, P K_{I D_{i}}, l_{i}, l_{i} b P\right)$. If such a tuple is found, $\mathcal{C}$ answers $l_{i} b P$. Otherwise, $\mathcal{C}$ chooses a random value $l \in Z_{q}$, puts the $\left(U_{i}, V_{i}, I D_{i}, P K_{I D_{i}}, l, l b P\right)$ into $L_{4}$, and returns $l b P$.

$H_{2}$ queries: For each new query $\left(U_{i}, T_{i}, R_{i}, I D_{i}, P K_{I D_{i}}\right), \mathcal{C}$ proceeds as follows:

1. It checks if $\hat{e}(a P, b P)=\hat{e}\left(P, R_{i}\right)$. If so, $\mathcal{C}$ returns $R_{i}$ and stops.

2. $\mathcal{C}$ goes through the list $L_{2}$ looking for entries $\left(U_{i}, T_{i}, \star, I D_{i}, P K_{I D_{i}}, h_{i}\right)$ such that $\hat{e}\left(U_{i}, P K_{I D_{i}}\right)=$ $\hat{e}\left(P, R_{i}\right)$. If such a tuple exists, it returns $h_{i}$ (and replaces the symbol $\star$ with $R_{i}$ ).

3. If $\mathcal{C}$ reaches this point of execution, it returns a random $h$ and updates the list $L_{2}$, which is initially empty, with a tuple containing the input and return values.

Symmetric key generation queries: Let $I D_{s}, I D_{r}$ be the identity of the sender and that of the receiver respectively used by $\mathcal{F}$ in a symmetric key generation query. For each new query $\left(I D_{s}, I D_{r}\right)$, $\mathcal{C}$ proceeds as follows:

1. If $I D_{s} \neq I D_{j}, \mathcal{C}$ computes the private key $S_{I D_{s}}$ corresponding to $I D_{s}$ by running the private key extraction query algorithm. Then $\mathcal{C}$ runs $(K, \omega) \leftarrow \operatorname{Sym}\left(\right.$ params, $\left.S_{I D_{s}}, I D_{s}, P K_{I D_{s}}, I D_{r}, P K_{I D_{r}}\right)$ and sends $K$ to $\mathcal{F}$. Note that $\mathcal{C}$ needs to store $\omega$ and to overwrite any previous value.

2. If $I D_{s}=I D_{j}$ (and hence $I D_{r} \neq I D_{j}$ ), $\mathcal{C}$ chooses $u, v \in Z_{q}^{*}$, sets $U \leftarrow v a P$, and computes $T \leftarrow \hat{e}\left(U, D_{I D_{r}}\right)\left(\mathcal{C}\right.$ could computes $D_{I D_{r}}$ because it knows the master secret key $\left.s\right)$. Note that the $\omega$ is $\left(u, v, U, I D_{s}, P K_{I D_{s}}, I D_{r}, P K_{I D_{r}}\right)$ in this case.

3. It goes through list $L_{2}$ looking for an entry $\left(U, T, R, I D_{r}, P K_{I D_{r}}, h\right)$ for some $R$ such that $\hat{e}\left(U, P K_{I D_{r}}\right)=\hat{e}(P, R)$, where $P K_{I D_{r}}$ is obtained by calling the request public key oracle on $I D_{r}$. If such an entry exists, it computes $K \leftarrow h$. Otherwise it uses a random $h$ and updates the list $L_{2}$ with $\left(U, T, \star, I D_{r}, P K_{I D_{r}}, h\right)$.

Key encapsulation queries: $\mathcal{F}$ produces a arbitrary tag $\tau$. $\mathcal{C}$ checks whether there exists a stored value $\omega$. If there is not, it returns $\perp$ and terminates. Otherwise $\mathcal{C}$ proceeds as follows.

1. If $I D_{s} \neq I D_{j}, \mathcal{C}$ answers the query by a call to $\operatorname{Encap}(\omega, \tau)$.

2. If $I D_{s}=I D_{j}$ (and $\left.I D_{r} \neq I D_{j}\right), \mathcal{C}$ defines the hash value $H_{3}\left(U, \tau, I D_{s}, P K_{I D_{s}}\right)$ as $H \leftarrow$ $v^{-1}\left(u P-H_{4}\right)$, where $H_{4}$ is the output of $H_{4}\left(U, \tau, I D_{s}, P K_{I D_{s}}\right)$. If a such a hash queries has been responded with a different value before, it aborts the simulation. This means that $\mathcal{C}$ updates list $L_{3}$ with tuple $\left(U, \tau, I D_{s}, P K_{I D_{s}}, \perp, H\right)$. Finally, $\mathcal{C}$ sets $W=D_{I D_{s}}+$ uaP. $\mathcal{C}$ returns $\psi \leftarrow(U, W)$. 
Key decapsulation queries: For a key decapsulation query on a $\left(\psi^{\prime}, \tau^{\prime}\right)$ for identities $I D_{s}$ and $I D_{r}, \mathcal{C}$ proceeds as follows.

1. It executes the verification part of the decapsulation algorithm obtaining $Q_{I D_{s}}$ and $P K_{I D_{s}}$ by calling $H_{1}$ and request public key oracles. It returns $\perp$ if the verification does not succeed.

2. It checks if $I D_{s}=I D_{j}$ and if this is the case then $\mathcal{C}$ can solve the $\mathrm{CDH}$ problem as described below.

3. It calculates $T=\hat{e}\left(U, e_{r} P_{p u b}\right)$, where $\left(I D_{r}, e_{r}\right)$ is obtained from $H_{1}$.

4. If $I D_{r} \neq I D_{j}$, it computes $R \leftarrow x_{I D_{r}} U$, where $x_{I D_{r}}$ is obtained (and hence $P K_{I D_{r}}$ ) from either the adversary or by calling the request public key oracle. Then $\mathcal{C}$ returns $K \leftarrow H_{2}\left(U, T, R, I D_{r}, P K_{I D_{r}}\right)$.

5. If $I D_{r}=I D_{j}$, the correct value of $R$ cannot be computed. To return a consistent answer, $\mathcal{C}$ goes through $L_{2}$ and looks for a tuple $\left(U, T, R, I D_{r}, P K_{I D_{r}}, h\right)$, for different values of $R$, such that $\hat{e}(U, a P)=\hat{e}(P, R)$. If such an entry exists, the correct value of $R$ is found and returns $K \leftarrow h$.

6. If $\mathcal{C}$ reaches this point of execution, it places the entry $\left(U, T, \star, I D_{r}, P K_{I D_{r}}, h\right)$ for a random $h$ on list $L_{2}$ and returns $K \leftarrow h$. The symbol $\star$ denotes an unknown value of $R$.

Finally, $\mathcal{F}$ outputs a produces a quaternion $\left(\tau^{*}, \psi^{*}, I D_{s}^{*}, I D_{r}^{*}\right) \cdot \mathcal{C}$ checks if $I D_{s}^{*}=I D_{j}$. If not, it aborts execution. Otherwise, it obtains $P K_{I D_{s}^{*}}$ by calling the request public key oracle on $I D_{s}^{*}$ and retrieves $t^{*}$ and $l^{*}$ from lists $L_{3}$ and $L_{4}$ by querying $H_{3}$ and $H_{4}$ on $\left(U^{*}, \tau^{*}, I D_{s}^{*}, P K_{I D_{s}^{*}}\right)$. Note that if $\mathcal{C}$ succeeded, then the verification condition holds:

$$
\begin{aligned}
& \hat{e}\left(P, W^{*}\right)=\hat{e}\left(P_{p u b}, Q_{I D_{s}^{*}}\right) \hat{e}\left(U^{*}, H^{*}\right) \hat{e}\left(P K_{I D_{s}^{*}}, H^{\prime *}\right) \\
& \hat{e}\left(P, W^{*}\right)=\hat{e}\left(P_{p u b}, Q_{I D_{s}^{*}}\right) \hat{e}\left(U^{*}, t^{*} P\right) \hat{e}\left(a P, l^{*} b P\right) \\
& \hat{e}\left(P, l^{*} a b P\right)=\hat{e}\left(P, W^{*}-D_{I D_{s}^{*}}-t^{*} U^{*}\right)
\end{aligned}
$$

and thus $\mathcal{C}$ can compute

$$
a b P=\left(W^{*}-D_{I D_{s}^{*}}-t^{*} U\right) / l^{*} .
$$

Let us now analyze the probability that $\mathcal{C}$ succeeds in solving the $\mathrm{CDH}$ problem instance. For this to happen, the simulation must run until the end of the game, the adversary must pick a specific identity as $I D_{j}^{*}$, and it must query the hash functions $H_{3}$ and $H_{4}$ to properly construct the forgery. The probability that $\mathcal{F}$ is able to produce a forgery without querying both hash functions is upper bounded by $2 / 2^{k}$.

The probability that $\mathcal{C}$ aborts the simulation is related with the following events:

$-\mathcal{F}$ places a full private key extraction on $I D_{j}$.

$-\mathcal{C}$ wants to simulate a key encapsulation query and this leads to an inconsistency in the $H_{3}$ simulation.

Note that if $\mathcal{F}$ places the first fatal query, then it could not possibly use $I D_{j}$ as the sender identity in the forgery it produces at the end of the game, so we can pinpoint the probability that $\mathcal{C}$ does not abort the simulation due to these events and $\mathcal{F}$ picks the only useful case for solving $\mathrm{CDH}$ as $1 / q_{T}$. Note that the maximum length of the list $L_{K}$ is $q_{T}$, as stated in the Lemma

The latter fatal event occurs if $\mathcal{C}$ 's simulation triggers a collision in its simulation of $H_{3}$. Since the maximum size of $L_{3}$ is $q_{S K}+q_{D}+q_{3}+1$, we can upper bound the probability that this occurs as $q_{S K}\left(q_{S K}+q_{D}+q_{3}+1\right) / 2^{k}$. The result follows. 